ARTICLE OPEN

\title{
TCR-pMHC bond conformation controls TCR ligand discrimination
}

Dibyendu K. Sasmal ${ }^{1}$, Wei Feng ${ }^{1}$, Sobhan Roy ${ }^{2}$, Peter Leung $\mathbb{D}^{1}$, Yanran He ${ }^{3}$, Chufan Cai ${ }^{1}$, Guoshuai Cao (D) ${ }^{1}$, Huada Lian ${ }^{4}$, Jian Qin ${ }^{5}$, Enfu Hui ${ }^{6}$, Hans Schreiber ${ }^{3}$, Erin J. Adams ${ }^{2}$ and Jun Huang (iD ${ }^{1}$

A major unanswered question is how a TCR discriminates between foreign and self-peptides presented on the APC surface. Here, we used in situ fluorescence resonance energy transfer (FRET) to measure the distances of single TCR-pMHC bonds and the conformations of individual TCR-CD3 receptors at the membranes of live primary $T$ cells. We found that a TCR discriminates between closely related peptides by forming single TCR-pMHC bonds with different conformations, and the most potent pMHC forms the shortest bond. The bond conformation is an intrinsic property that is independent of the binding affinity and kinetics, TCR microcluster formation, and CD4 binding. The bond conformation dictates the degree of CD3 3 dissociation from the inner leaflet of the plasma membrane via a positive calcium signaling feedback loop to precisely control the accessibility of CD3 ITAMs for phosphorylation. Our data revealed the mechanism by which a TCR deciphers the structural differences among peptides via the TCR-pMHC bond conformation.

Keywords: Bond conformation; T cell receptor; Single molecule FRET; ligand discrimination

Cellular \& Molecular Immunology (2020) 17:203-217; https://doi.org/10.1038/s41423-019-0273-6

\section{INTRODUCTION}

A TCR consists of a variable TCRaß heterodimer and a nonvariable transmembrane signal transduction CD3 complex containing $\mathrm{CD} 3 \gamma \varepsilon$ and $\mathrm{CD} 3 \delta \varepsilon$ heterodimers and a $\mathrm{CD} 3 \zeta \zeta$ homodimer. TCRs specifically and sensitively detect a small number of agonist pMHCs among a plethora of structurally similar self-pMHCs to trigger antigen-specific immune responses. ${ }^{1-3}$ Despite intense efforts, the mechanism underlying TCR ligand discrimination remains a major unanswered question in immunology. ${ }^{4-6}$ TCR ligand discrimination is uniquely challenging. First, it requires TCRs to detect very rare foreign pMHCs in the presence of considerably abundant self-pMHCs. Second, it requires proper signaling propagation from surface TCR binding to induce intracellular CD3 phosphorylation. Although it is generally thought that the engagement of the extracellular TCRa $\beta$ domain with a pMHC results in biochemical changes in the cytoplasmic portions of the CD3 complex, there are no experimental data that can directly depict this process with enough spatiotemporal resolution at the membranes of live primary $\mathrm{T}$ cells.

Many models have been proposed to explain the molecular mechanism underlying TCR discrimination. The TCR conformational change model postulates that a conformational change in a TCR occurs upon pMHC binding, but no conformational changes at the TCR-pMHC binding interface have been identified that are conserved in TCR-pMHC crystal structures. ${ }^{5,7}$ However, crystal structures only provide a "snapshot" of the thermodynamically stable conformations of purified TCR and pMHC proteins.
The TCR is an active molecular machine that is anchored at the live cell membrane, associates with CD3 signaling units, links to the cytoskeleton, and interacts with other signaling molecules. "Active" TCR molecules at cell membrane are very different from purified "quiescent" TCR proteins isolated from the cellular environment. A typical example of this is that the in situ binding kinetics and affinities of TCR-pMHC interactions measured at the T-cell membrane are dramatically different from those measured in vitro in solution. ${ }^{3,8}$ It has long been speculated that the TCR at the cell membrane undergoes conformational changes upon pMHC binding. This hypothesis is attractive, but it has never been experimentally proven at the membranes of live primary $\mathrm{T}$ cells, mainly due to the lack of appropriate experimental approaches. Furthermore, another key issue for TCR discrimination is how different TCR-pMHC interactions result in distinct biochemical changes in the cytoplasmic domains of $\mathrm{CD} 3$. It has been suggested that CD3 ITAMs are sequestered in the plasma membrane and TCR-pMHC engagement pulls the CD3 cytoplasmic domains away from the membrane, thus making the ITAMs accessible to phosphorylation by Lck. ${ }^{9}$ However, other studies argue that the release of ITAMs from the plasma membrane is unlikely to be a prerequisite step in the initiation of TCR signaling. ${ }^{6}$

To carefully examine possible TCR conformational changes and investigate the molecular mechanism of TCR triggering in situ, we used fluorescence resonance energy transfer (FRET), ${ }^{10,11}$ which functions as a spectroscopic ruler with subnanometer precision, to

\footnotetext{
${ }^{1}$ The Pritzker School of Molecular Engineering, The University of Chicago, Chicago, IL, USA; ${ }^{2}$ Department of Biochemistry and Molecular Biology, The University of Chicago,

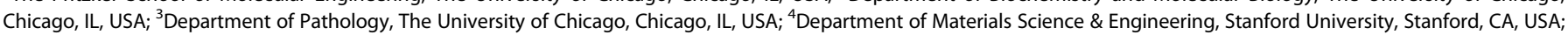

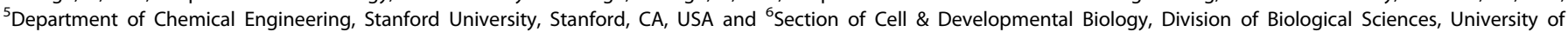
California, San Diego, CA, USA

Correspondence: Jun Huang (huangjun@uchicago.edu)
}

Received: 3 August 2019 Accepted: 7 August 2019

Published online: 17 September 2019 

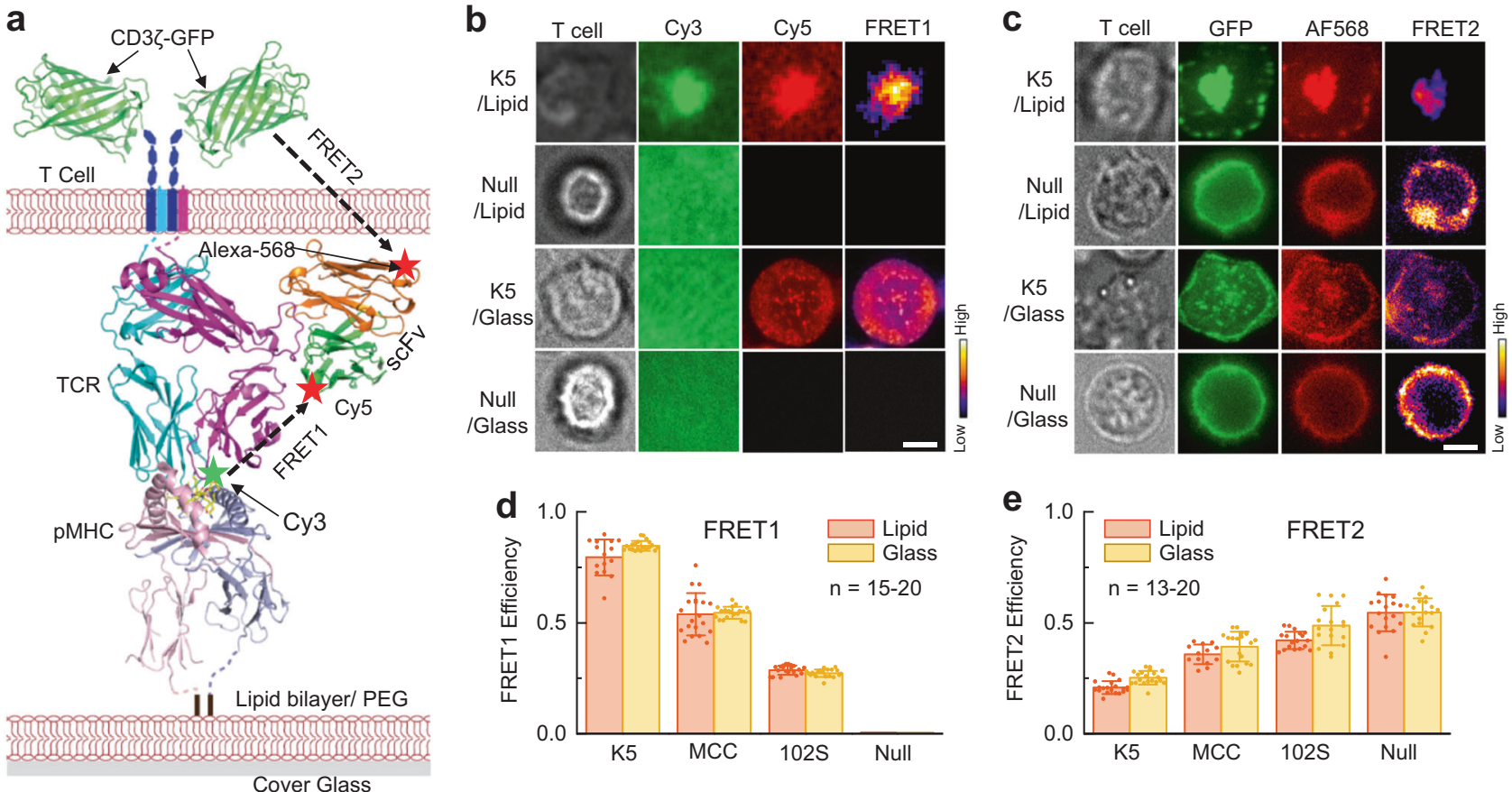

Fig. 1 Measurement of TCR conformational dynamics by FRET. a A composite structural model of 5C.C7 TCR (PDB ID: 4P2R), MCC-IE ${ }^{k}$ (PDB ID: 3QIU), scFv (PDB ID: 1NFD), and CD3 $\zeta$-GFP (PDB ID for GFP: 1GFL). To measure the TCR-pMHC bond conformational dynamics using FRET1, the TCR was labeled by Cy5 via the scFv J1, and the peptide C-terminus was labeled by Cy3. For determining the TCR-CD3 $C$ conformational changes by FRET2, the TCR was labeled by Alexa Fluor 568 (Alexa568) via scFv J3, and the CD3 $\zeta$ C-terminus was tagged with GFP. Extracellular Cy3/Cy5 FRET1 and transmembrane GFP/Alexa568 FRET2 are indicated by dashed lines. The pMHC molecules were anchored on either a lipid bilayer or on a PEG-Ni ${ }^{2+}$ glass surface (Fig. S1a). Note: J1 and J3 are different scFvs, and each has only one unique labeling site. b, c T cell, donor, acceptor, and calculated FRET signals of TCRs interacting with K5 or a null pMHC on a lipid bilayer or on a glass surface. The calculated FRET efficiency images for FRET1 (b) and FRET2 (c) are shown in pseudocolor; the cold-to-hot color spectrum represents weak-to-strong FRET efficiency. Representative data from 3-5 independent experiments for each PMHC at $37^{\circ} \mathrm{C}$ are shown. The scale bar is $5 \mu \mathrm{m}$. d, e FRET efficiencies measured for K5, MCC, 102S, and null pMHCs on a lipid bilayer (red) and a glass surface (yellow) for extracellular TCR-pMHC FRET1 (d) and transmembrane TCR-CD3 $\zeta$ FRET2 (e). At least 13 cells were used to determine the FRET efficiency for each pMHC. Also see Figs. S1-4 and Movies S1-3

measure the intermolecular distance of a TCR-pMHC bond and the intramolecular conformation of a TCR-CD3 complex at the immunological synapse of a live primary $\mathrm{CD}^{+} \mathrm{T}$ cell in real time with high spatiotemporal resolution. These experiments enabled us to critically test the TCR conformational change model and probe the molecular mechanism underlying TCR ligand discrimination.

\section{RESULTS}

\section{FRET design}

To determine the conformation of a single TCR-pMHC bond, a TCR and a pMHC were site-specifically labeled with the FRET acceptor Cy5 and the FRET donor Cy3, respectively. ${ }^{8}$ The peptide within the MHC molecule was labeled with $\mathrm{Cy} 3$, and the TCR was labeled with the anti-TCR single-chain variable fragment (scFv) J1-Cy5 (Fig. 1a and Fig. S1a). The intermolecular distance between Cy3 and $\mathrm{Cy} 5$ provided a reasonable approximation of the conformation (or compactness) of the TCR-pMHC bond. The conformation of a single TCR-pMHC bond on the cell surface was measured by Cy3/Cy5 FRET (FRET1) (Fig. 1a) in real time by total internal reflection fluorescence (TIRF) microscopy (Fig. S1b). The Cy3/Cy5 inter-dye distance of a TCR-pMHC bond is denoted as the TCR-pMHC bond distance to describe the TCR-pMHC bond conformation in the following paragraphs. To measure the intramolecular distance between the TCR and CD3 3 in a transmembrane TCR-CD3 $\zeta$ complex, we added a green fluorescent protein (GFP) to the $\mathrm{C}$-terminus of the $\mathrm{CD} 3 \zeta$ chain, and the TCR was labeled with an Alexa Fluor 568 fluorophore using a different anti-TCR SCFv J3 with a unique labeling site close to the cell membrane. ${ }^{8}$ The real-time intramolecular distances of the TCR-CD3 Complexes were measured by GFP/Alexa Fluor 568 FRET (FRET2) (Fig. 1a) using epifluorescence time-lapse microscopy (Fig. S1b).

We first performed experiments to assess the feasibility and specificity of cell surface FRET1 and transmembrane FRET2 on a lipid bilayer (Fig. 1a) and on a glass surface (Fig. S1a) containing the PMHC and accessory molecules ICAM-1 and B7-1, respectively (Fig. S2 and Movie S1-3). For cell surface FRET1, we readily detected FRET signals from three agonist pMHCs but not from a null pMHC on the lipid bilayer and on the glass surface, and the FRET efficiencies $\left(E_{\mathrm{FRET}}\right)$ were positively correlated with the pMHC potencies in activating $T$ cells in vitro ${ }^{12}$ (Fig. $1 \mathrm{~b}, \mathrm{~d}$ ). The average synaptic $E_{\text {FRET } 1}$ was $0.79,0.54$, and 0.29 for the super agonist K5, the agonist MCC, and the weak agonist 102S, respectively. However, no synaptic FRET was observed for the null pMHC. These data validated the specificity of the cell surface TCR-pMHC FRET and were consistent with the results of a previous report. ${ }^{8}$ In contrast, the transmembrane TCR-CD3 $\zeta$ FRET2 efficiencies were inversely correlated with the $\mathrm{PMHC}$ potencies, and the highest FRET was observed for the null pMHC (Fig. 1c, e). In the presence of the K5, MCC, and $102 \mathrm{~S}$ pMHCs, the transmembrane FRET change was only detected in the TCR-CD3 3 colocalized microclusters and not outside of the colocalized microclusters (Fig. 1c and Fig. S3a). Replacing the FRET acceptor Alexa Fluor 568 with a Cy5 dye abolished the transmembrane FRET2 (Fig. S3b). These experiments confirmed the specificity of the transmembrane TCR-CD3ろ FRET. 
Measurement of the TCR-pMHC bond conformation by smFRET We next performed Cy3/Cy5 single-molecule FRET (smFRET) on a lipid bilayer to measure the conformations of single TCR-pMHC bonds using TIRF microscopy (Fig. 2 and Fig. S1b). TCR-pMHC bond formation at live T-cell membrane brought the donor Cy3 and the acceptor $\mathrm{Cy} 5$ into close enough proximity to produce smFRET (Figs. 1a and 2a). The fluorescent intensities of Cy3 and Cy5 were simultaneously recorded in real time (Fig. 2b, top panel, and Fig. S4a), and the $E_{\mathrm{FRET}}$ values were calculated based on the fluorescence intensities of the donor Сy3 and the acceptor Cy5 (Fig. 2b, bottom panel). As $E_{\mathrm{FRET}}$ is inversely proportional to the distance between the donor and the acceptor to the sixth power, Cy3/Cy5 smFRET serves as a sensitive microscopic ruler to precisely measure the intermolecular TCR-pMHC bond distance in real time during binding. The time trajectory of $E_{\mathrm{FRET}}$ showed that the TCR-pMHC bond is dynamic and exhibits continuous conformational changes (Fig. 2b), which is in line with the results in recent reports that demonstrated that a TCR or a pMHC undergoes conformational changes upon binding. ${ }^{13,14}$ The recording of the fluctuating conformational trajectories provided for the real-time observation of single TCR-pMHC bond dynamics. By plotting the $E_{\mathrm{FRET}}$ histogram and fitting it with a Gaussian function, we determined the most probale $E_{\mathrm{FRET}}$ value of 0.7 , corresponding to a $47 \pm 3 \AA$ distance between the 5C.C7 TCR and the super agonist $\mathrm{K} 5 \mathrm{pMHC}$ in a representative smFRET trajectory (Fig. 2c). We repeated our single-bond measurements for the K5 pMHC and performed smFRET experiments for the agonist MCC and weak agonist $102 \mathrm{~S}$ pMHCs (Fig. S5). After collecting many individual smFRET trajectories for each PMHC, we pooled all $E_{\mathrm{FRET}}$ data together and plotted the histograms for each pMHC (1577-1933 trajectories per histogram, Fig. 2d). Remarkably, the distributions of $E_{\mathrm{FRET}}$ showed that the intermolecular TCR-pMHC bond distances were peptide dependent, and single TCR-pMHC bonds were highly dynamic within a continuous range of conformational states. Fitting each histogram with a Gaussian function (curves, Fig. 2d) yielded the most probable intermolecular TCR-pMHC bond distance for each pMHC: $44 \pm 9 \AA$ for K5 (super agonist), $54 \pm 11 \AA$ for MCC (agonist), and $66 \pm 18 \AA$ for $102 \mathrm{~S}$ (weak agonist) (Fig. 2d), directly revealing the angstromlevel, binding-induced, peptide-dependent TCR-pMHC bond distances and conformational dynamics in situ. This key information was missing from previously reported TCR-pMHC crystal structures. ${ }^{7,15}$ In addition, we experimentally verified that the differences in the distances of the pMHCs were not due to peptide labeling-derived noise (Fig. S6). We further measured the average TCR-pMHC bond distances using ensemble FRET on both a lipid bilayer and a glass surface (Figs. S4b and S7), and the results confirmed the single-bond measurements obtained using smFRET (Fig. 2d).

We then quantified the binding strength of each single TCR-pMHC bond by analyzing the potential-of-mean-force (PMF), ${ }^{16}$ which measures the free energy cost of the variation in bond conformation. PMF is at minimum when the bond conformation is at equilibrium, and the PMF curve indicates the sizes of the fluctuations (Fig. 2e). Clearly, the super agonist K5 and agonist MCC have deep and narrow energy wells, indicating their strong and stable bonds. In contrast, the weak agonist $102 \mathrm{~S}$ has a shallow and wide energy well, suggesting its weak bond strength and unstable binding state. Overall, the depth and width of the PMF curve revealed that K5 and MCC form more stable (shorter) bonds with TCRs compared with 102S (Fig. 2e), which is consistent with previous reports that indicated that $\mathrm{K} 5$ and $\mathrm{MCC}$ have higher $3 \mathrm{D}$ in vitro binding affinities with TCRs than $102 S^{8,12}$ and that TCR triggering is dependent on the receptor-ligand complex dimensions. ${ }^{17,18}$ Our smFRET measurements not only revealed that TCR triggering is critically dependent on the conformation of a TCR-pMHC bond but also linked the bond conformation to the bond energy, thus providing a fundamental basis for understanding TCR ligand discrimination.

TCR-pMHC bond conformation is an intrinsic property independent of binding kinetics and affinity

Cell surface smFRET is highly distance dependent ${ }^{8}$ and only produces a FRET signal when a TCR binds to a pMHC (Fig. 1b, d, comparison of $\mathrm{K} 5$ with null). To confirm the bound state, we tracked and compared the diffusion of single pMHCs in smFRET and those of individual-free pMHCs on the lipid bilayer (Fig. $2 \mathrm{f}, \mathrm{g}$ and Fig. S8). We found that the pMHCs in smFRET were tightly restricted within the synapse, whereas the free pMHCs were very mobile, as shown by their distinct diffusion trajectories and coefficients (Fig. 2f, top panel). The diffusion coefficient of a pMHC in smFRET was close to 0 , which was 140 -fold smaller than that of a free pMHC (Fig. 2f, bottom panel). We further plotted the horizontal $(x)$ and vertical $(y)$ positions of single-tracked pMHCs over time (Fig. 2g, top panel) and analyzed the position changes according to the 3-point moving standard deviation (Fig. 2g, bottom panel). The pMHCs in smFRET and the free pMHCs on the lipid bilayer showed dramatic differences in diffusion at the singlemolecule level. These differences have been previously used to differentiate TCR-bound pMHCs from unbound pMHCs for kinetic measurements. ${ }^{19,20}$ Our tracking analyses verified that we detected a FRET signal only if a TCR was bound to a pMHC. In other words, smFRET only measured the conformational dynamics of single TCR-pMHC bonds during the bound state, which are independent of those of unbound molecules and bond association/dissociation. Thus, our conformational measurements by smFRET were independent of TCR-pMHC binding affinity and kinetics.

The effects of TCR microclusters and CD4 binding

TCRs form microclusters upon antigen stimulation (Movie S4). To measure the conformational dynamics of single TCR-pMHC bonds without possible interference from TCR microclusters, we used latrunculin $A(L A)$ to prevent the formation of TCR microclusters during smFRET measurements. Consistent with the results of a previous study, no TCR microclusters were observed at the T-cell synapse after treatment with LA. ${ }^{21} \mathrm{We}$ then performed smFRET in the absence of TCR microclusters. We found that the most probale TCR-pMHC bond distances (and distributions) measured in the absence of TCR microclusters were very close to those measured in the presence of TCR microclusters (compare Fig. $2 \mathrm{~d}$ with Fig. 3), suggesting that our TCR-pMHC bond distance measurements were not affected by TCR microclusters. These results further confirmed that we had determined the true intermolecular TCR-pMHC bond distances at the single-molecule level.

Because the CD4 coreceptor also binds to $\mathrm{PMHC}$, to evaluate the effect of CD4 binding on the TCR-pMHC bond distances, we used an antibody to block CD4 binding to pMHCs and performed smFRET measurements. Our data showed that CD4 binding had little effect on the TCR-pMHC bond distances (compare Fig. $2 \mathrm{~d}$ and Fig. 3b), which is consistent with the results of previous kinetic studies showing that CD4-pMHC binding is negligible compared to TCR-pMHC interaction in vitro and in situ. ${ }^{8,22-24}$

We then determined the most probable TCR-pMHC bond distances for K5 (super agonist), MCC (agonist), and 102S (weak agonist) under different conditions by smFRET and bulk FRET, as shown in Fig. 3c. We found that neither the disruption of TCR microclusters nor the blocking of CD4 binding changed the TCR-pMHC bond conformation for the three pMHCs. These data (Fig. 3) significantly confirmed the aforementioned smFRET measurements that were made in the absence of any treatments (Fig. 2) and further strengthened our conclusion that the single TCR-pMHC bonds of the three pMHCs have different conformations at the single-molecule level. 

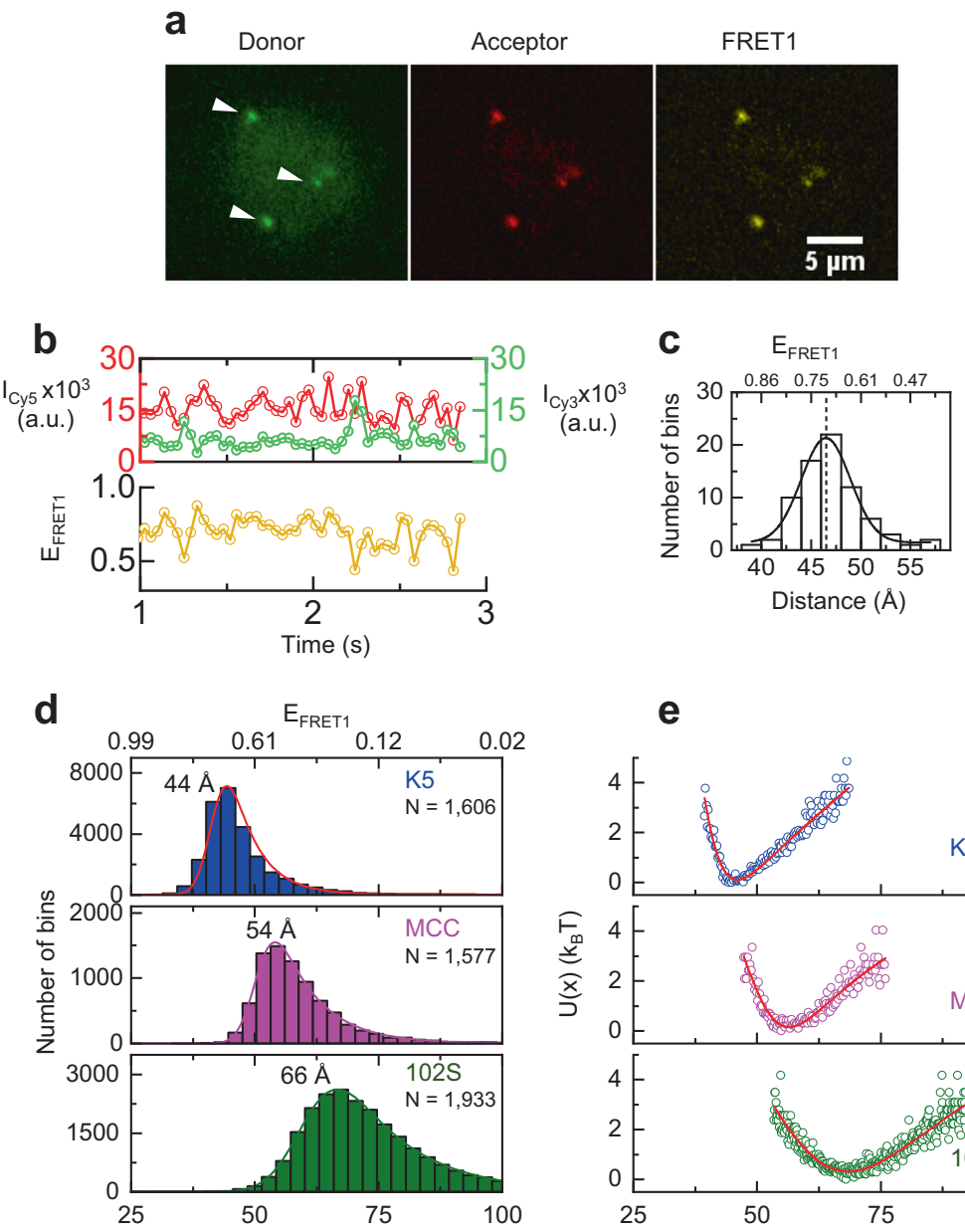

e
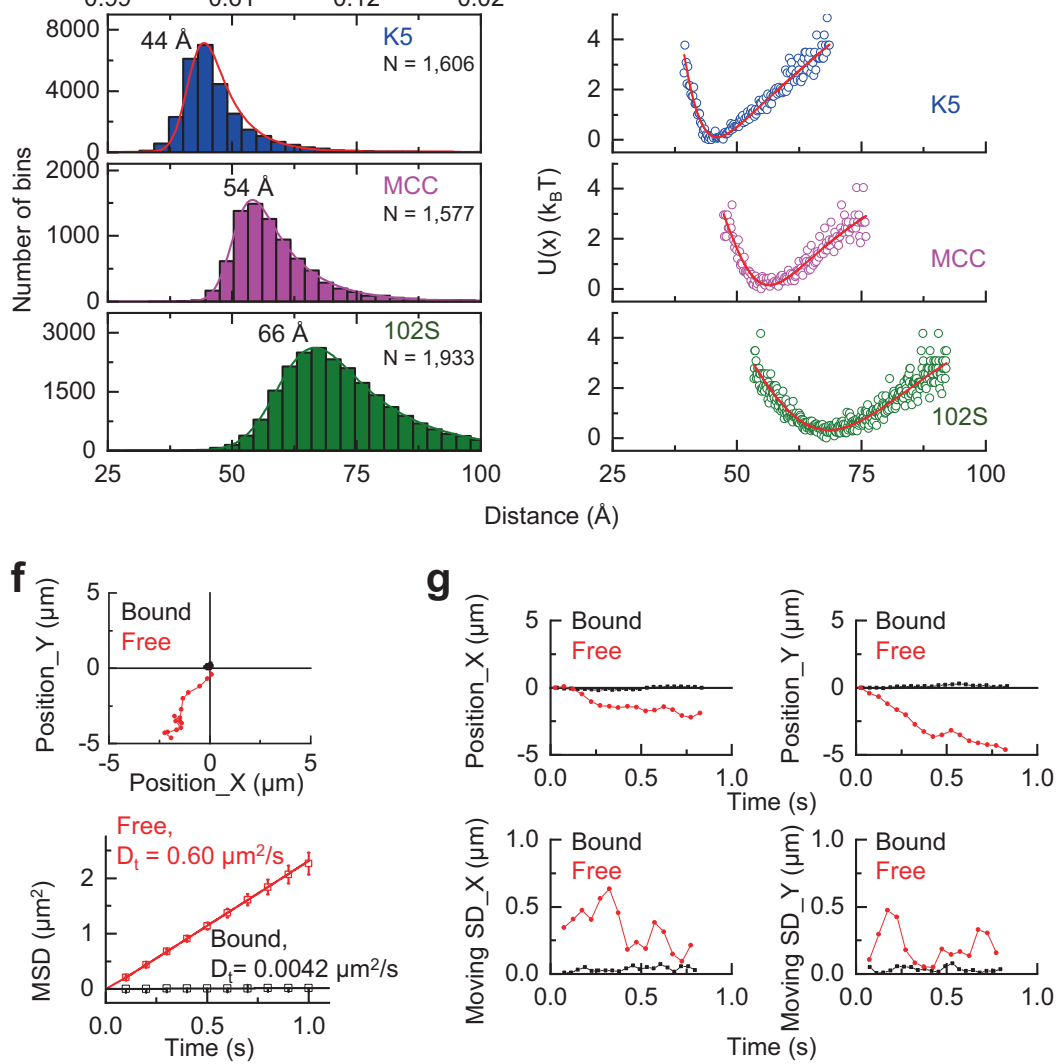

Fig. 2 The conformational dynamics of single TCR-pMHC bonds. a A representative smFRET event mediated by the interaction between a $5 C$. C7 TCR and a K5 pMHC. The donor, acceptor, and FRET signals are shown, and white arrows indicate single molecules. b Single-molecule time trajectories of the donor (green, Cy3-pMHC) and the acceptor (red, Cy5-TCR) intensities (upper panel) and the corresponding time trajectory of smFRET efficiency (yellow, lower panel). Single donor and acceptor molecules were tracked in real time to calculate the FRET efficiency. Also see Fig. S5. c Histogram of the Cy3-Cy5 distances that was calculated from the smFRET efficiencies shown b (lower panel, see methods) and fitted according to a Gaussian distribution (black curve). Also see Fig. S6. d Histograms of the TCR-pMHC bond distances for a single bond for the K5, MCC, and 102S pMHCs. Each histogram used 1577-1933 trajectories to determine the most probale TCR-pMHC bond distance for each pMHC. Also see Figs. S7 and S8. e PMF determination of single TCR-pMHC bonds for the K5, MCC, and 102S pMHCs. PMF indicates the free energy changes as a function of the TCR-pMHC bond distance. $f$ Representative diffusion trajectories of single pMHCs under free (red) and bound (black) conditions (top panel). The mean squared displacement (MSD) vs. time ( $t$ ) was plotted to show the mobility of single-tracked pMHCs ( $x$-axis: acquisition time in seconds; $y$-axis: MSD). The calculated diffusion coefficients $\left(D_{t}\right)$ were determined for each type of diffusion (bottom panel). g The horizontal positions $x$ and vertical positions $y$ of each single-tracked pMHC were plotted vs. time $t$ for representative single free (red) and bound (black) pMHCs (top panels). The corresponding sliding standard deviation $\sigma$ for three consecutive points according to the $x$ and $y$ positions were calculated to reveal the position changes of the single-tracked pMHCs (bottom panels) 


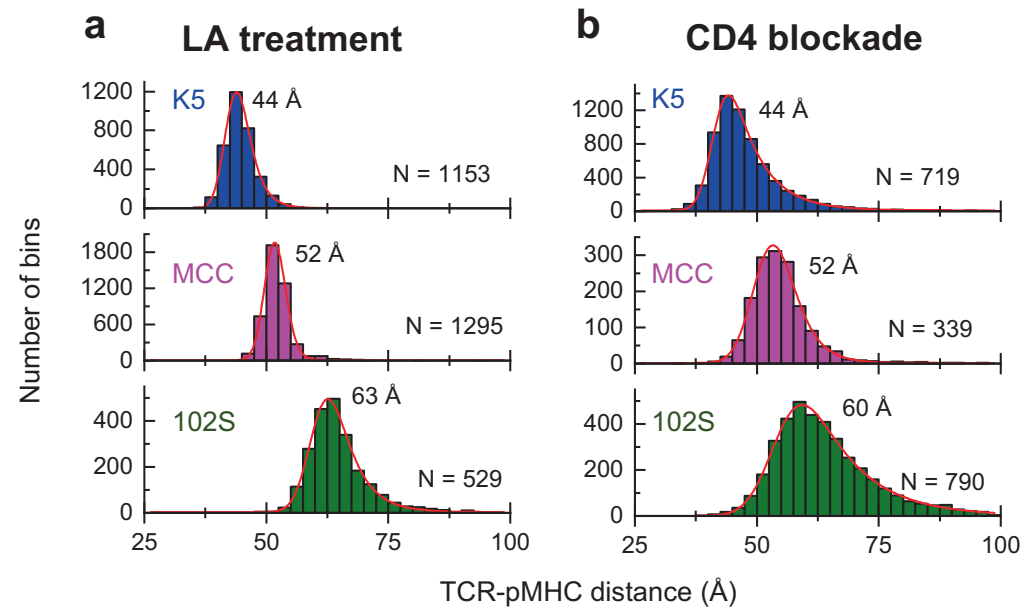

C TCR-pMHC bond distances

\begin{tabular}{llll}
\hline pMHC & $\mathrm{K} 5(\AA)$ & $\mathrm{MCC}(\AA)$ & $102 \mathrm{~S}(\AA)$ \\
\hline smFRET: no treatment & $44 \pm 9$ & $54 \pm 11$ & $66 \pm 18$ \\
smFRET: LA treatment & $44 \pm 3$ & $52 \pm 3$ & $63 \pm 7$ \\
smFRET: CD4 blockade & $44 \pm 9$ & $52 \pm 7$ & $60 \pm 12$ \\
Bulk FRET: no treatment & $43 \pm 4$ & $53 \pm 4$ & $63 \pm 1$
\end{tabular}

Fig. 3 The Effects of TCR microclusters and CD4 binding on TCR-pMHC bond conformation. smFRET TCR-pMHC bond distance measurements in the presence of the actin polymerization inhibitor latrunculin $A(L A)(1 \mu \mathrm{M})(\mathbf{a})$ or $10 \mu \mathrm{g} / \mathrm{mL}$ anti-CD4 blocking antibody (b) at $37^{\circ} \mathrm{C}$. In each case, cells were pretreated with LA or antibody for $1 \mathrm{~h}$ before introduction to the planar bilayer. Histograms of TCR-pMHC bond distances for the K5, MCC, and 102S pMHCs are shown. Each histogram used 339-1295 single TCR-pMHC bond trajectories to identify the most probable distance for each pMHC. c The TCR-pMHC bond distances for each pMHC in different conditions measured by FRET. Data are presented as the most probable distance \pm standard deviation (SD)

Measurement of intramolecular TCR-CD3 conformational changes by FRET

It is not clear what induces the dissociation of $\mathrm{CD} 3 \zeta$ from the membrane to initiate T-cell intracellular signaling. To further understand how surface TCR-pMHC bonds propagate extracellular recognition signals to intracellular $\mathrm{CD} 3 \zeta$ ITAMs across the cell membrane, we developed a transmembrane FRET assay to measure the conformational change between the extracellular TCR $\alpha \beta$ domain and the intracellular CD3 3 chain in the same TCR/ CD3Z complex (Fig. 1a). Upon the addition of 5C.C7 transgenic T cells with Alexa Fluor 568-labeled TCRs and GFP-tagged CD3 3 to a lipid bilayer containing pMHC ligands, we observed rapid microcluster formation and the instant production of transmembrane GFP/Alexa Fluor 568 FRET. The TCRs and CD3 3 were predominantly colocalized in the microclusters [Pearson correlation coefficient, ${ }^{25} 0.93 \pm 0.07$ ] (Fig. 4a, Figs. S9 and S10, and Movie S5), demonstrating the initiation of T-cell signaling via segregated TCR-pMHC bond-mediated close contacts. ${ }^{26}$ The high level of TCR-CD3Z colocalization suggested that the obligate assembly of TCR-CD3ろ was necessary for effective T-cell signaling and verified the specificity of transmembrane TCR-CD3 $\zeta$ FRET. TCRs and $\mathrm{CD} 3 \zeta$ microclusters continuously moved from the periphery to the center of the cell and merged into the immunological synapse (Fig. 4a). We tracked and measured the FRET efficiencies of each individual microcluster in real time. After converting the FRET efficiencies to TCR-CD3 3 distances, we plotted a three-dimensional figure (Fig. 4b) to illustrate the simultaneous lateral movement of TCR-CD3 3 complexes ( $x-y$ axis) (also see Fig. S10b) and the intramolecular TCR-CD3 changes across the cell membrane (z-axis) upon K5 pMHC engagement. The intramolecular TCR-CD3 $\zeta$ distances within different microclusters at equilibrium consistently showed an $\sim 15 \AA$ difference before and after K5 pMHC binding.
To test whether TCR-CD3 $\zeta$ conformational changes were dependent on TCR signaling, we used an Src kinase inhibitor PP2 ${ }^{27}$ to block TCR signaling. Consistent with the results of a previous study, ${ }^{21}$ PP2 completely abrogated T-cell calcium signaling (Fig. 4c, top), while TCRs could still form microclusters (Fig. 4c, bottom). To reveal the role of TCR signaling in TCR-CD3Z conformational changes, we plotted the time trajectories of the normalized intramolecular TCR-CD3 $\zeta$ distances against the stimulation time in the absence and presence of PP2 (Fig. 4d). In the absence of PP2, the intracellular $C D 3 \zeta$ chain quickly separated from the TCR extracellular domain upon TCR engagement with the $\mathrm{K} 5 \mathrm{pMHC}$ and then reached a stable, fully extended TCR-CD3 Conformation. In sharp contrast, the blocking of TCR signaling by PP2 completely abolished the TCR-CD3 conformational change (Fig. 4d). Consistently, this effect of TCR signaling was also found for the MCC and 102S pMHCs (Fig. S11). Together with the results of our negative control experiments (Fig. S12), these PP2 experiments (Fig. 4d and Fig. S11) further verified that transmembrane FRET was specifically caused by the TCR-CD3Z conformational change.

To compare the conformational dynamics induced by different pMHCs, we plotted the TCR-CD3 3 conformational changes against the stimulation time for the three pMHCs (Fig. 4e). We found that the TCR-CD3 Conformational change was dependent on the potency of the peptide, as demonstrated by the amplitude and speed of the TCR-CD3 $\zeta$ conformational change for different peptides (Fig. 4e). Compared with the nonstimulatory null peptide, the super agonist K5 caused the largest change, while the weak agonist $102 \mathrm{~S}$ caused the smallest conformational change in the TCR-CD3 Complex. Quantitatively, the K5, MCC, and 102S pMHCs resulted in $\sim 15 \AA, \sim 10 \AA$, and $\sim 5 \AA$ separations between the TCR and $\mathrm{CD} 3 \zeta$ after TCR-pMHC binding, respectively (Fig. 4e). We also found that TCR-CD3 conformational changes were restricted in the TCR/ CD3 $\zeta$ microclusters (Fig. 4a and Figs. S3a and S10a). Similar 

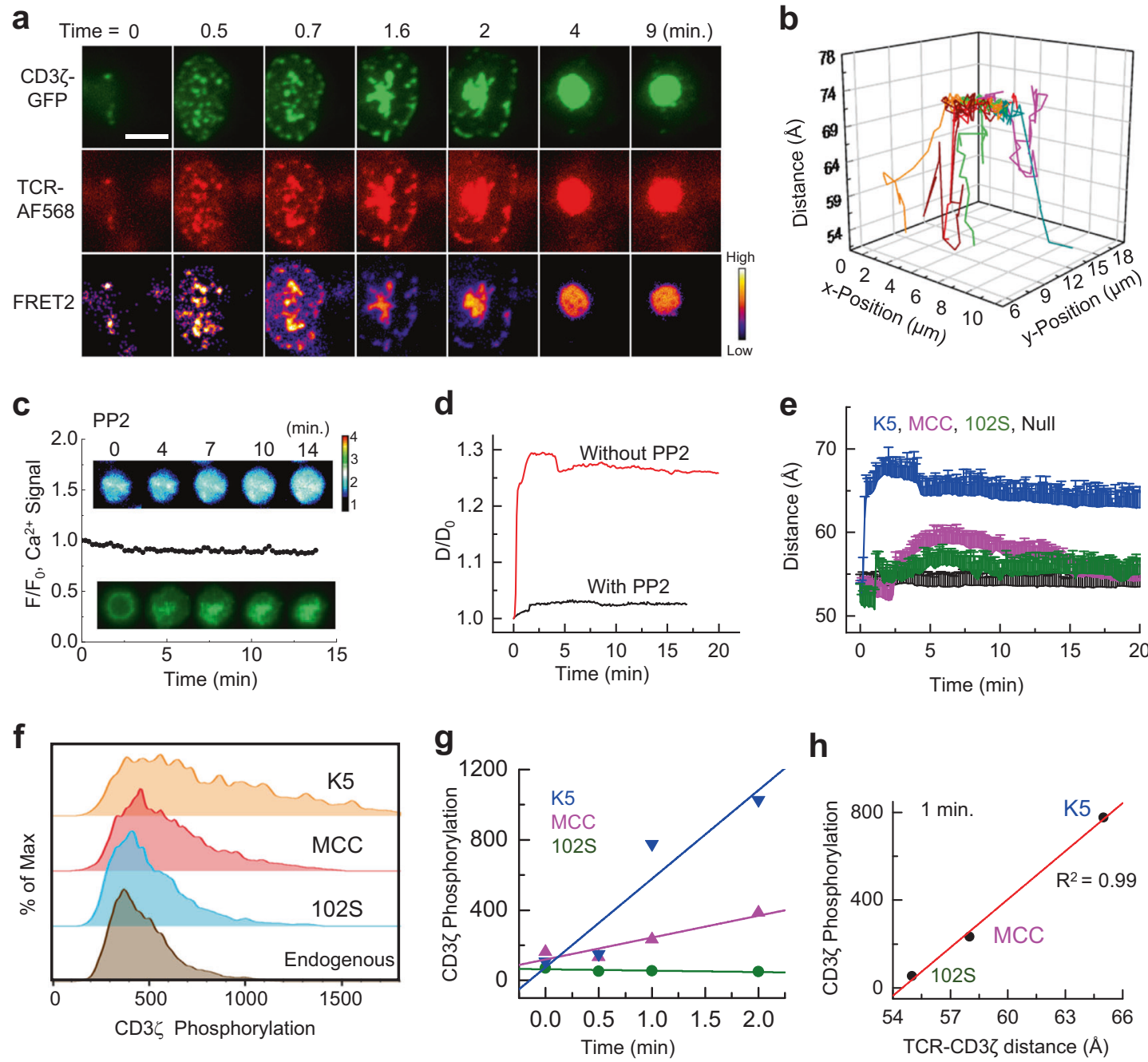

h

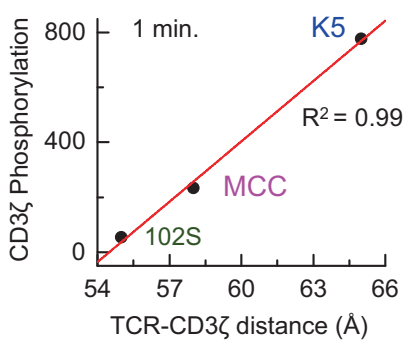

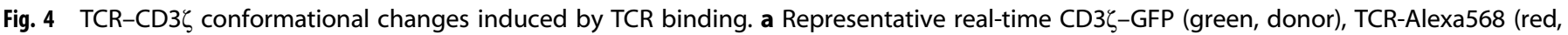
acceptor), and FRET (pseudocolor) signals from a typical transmembrane FRET experiment on a lipid bilayer at $37^{\circ} \mathrm{C}$ for the $\mathrm{K} 5 \mathrm{pMHC}$. The same experiments were performed for the MCC and 102S pMHCs. The calculated FRET2 efficiency is indicated in pseudocolor, with the coldto-hot color spectrum representing weak-to-strong FRET efficiency. Six to eight independent experiments were conducted for each pMHC. Scale bar: $5 \mu \mathrm{m}$. b 3D illustration of the lateral movement $(x-y)$ and intramolecular distance change $(z)$ between TCR and CD3 $\zeta$ in the individual $\mathrm{TCR} / \mathrm{CD} 3 \zeta$ microclusters shown in a on the lipid bilayer. The $x$ and $y$ positions of each microcluster were tracked by the TrackMate plugin in ImageJ, and the $z$ positions were calculated according to the corresponding FRET efficiencies. Also see Movie S4 and 5 for microcluster formation, Figs. S9 and S10 for data analysis, Fig. S18 for data calibration and Fig. S12 for the negative controls. c Time-lapsed microscopy

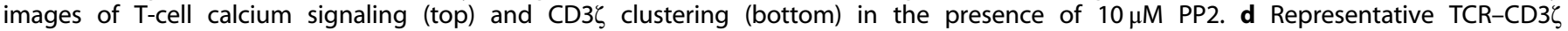
intramolecular distance changes with (black) and without (red) PP2 treatment. e Time-dependent TCR-CD3 $\zeta$ intramolecular distance changes upon TCR binding to the K5, MCC, 102S, or null pMHC. Each curve shows the average intramolecular distances at consecutive time points measured in three individual microclusters. f Phospho-flow cytometry showing the phosphorylation of CD3 $\zeta$ in T cells upon contact with antigen-presenting cells loaded with $\mathrm{K} 5, \mathrm{MCC}$, or $102 \mathrm{~S}$ peptide for $1 \mathrm{~min}$. $\mathbf{g}$ The time course of CD3 $\zeta$ phosphorylation upon stimulation with

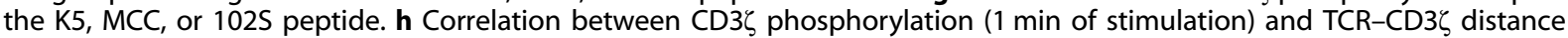

TCR-CD3ろ conformational changes were observed on the glass surface functionalized with pMHCs (Figs. S1a and S13). All together, these data highlighted that the TCR-CD3 conformational changes that occurred in the TCR microclusters were driven by TCR-pMHC engagement, which is consistent with previous reports that showed that TCR microclusters are hotspots for TCR signaling. ${ }^{2,21,28}$

We next examined how TCR-CD3 conformational changes regulate $\mathrm{CD} 3 \zeta$ phosphorylation. Of the three pMHCs tested, we found that the most potent, K5, caused the largest TCR-CD3 separation (Fig. 4e) and the highest level of phosphorylation (Fig. 4f, $g$ and Fig. S14). In contrast, the least potent, 102S, caused the smallest TCR-CD3 resulted in the lowest level of phosphorylation of $\mathrm{CD} 3 \zeta$ (Fig. 4f, $\mathrm{g}$ and Fig. S14). The $\mathrm{CD} 3 \zeta$ conformation and phosphorylation data together suggested that the TCR-CD3 conformation controls the degree of the dissociation of $\mathrm{CD} 3 \zeta$ from the inner leaflet of the plasma membrane, which influences the temporal (Fig. $4 \mathrm{~g}$ ) and spatial (Fig. 4h) aspects of the phosphorylation of the ITAMs in CD3C. Thus, transmembrane FRET could not only directly visualize TCR-CD3 Conformational changes in situ but could also explain the molecular mechanisms underlying signal propagation in TCR ligand discrimination.

Linking TCR conformational changes to T-cell responsiveness We then designed a series of experiments to test how the TCR conformation controls TCR binding, signaling, and activation. To test how the TCR-pMHC bond conformation regulates the TCR-pMHC interaction, we performed micropipette adhesion assays to measure the in situ two-dimensional (2D) TCR-pMHC binding kinetics and affinities (Fig. 5a-d, Table 1, Fig. S15, Table S1 
and Movie S6). ${ }^{3}$ Our data showed that the binding affinity (driven by the on-rate) is well correlated with the PMHC potency (Table 1). As revealed by the association of a shorter TCR-pMHC bond distance (Fig. 2d) with an increased TCR-pMHC binding affinity (Fig. 5b-d and Table 1), our data suggested that a shorter TCR-pMHC bond promotes the formation of a more stable TCR/ pMHC complex, which is consistent with the classic bond length theory in chemistry. ${ }^{29}$

To understand how the TCR-pMHC bond conformation triggers T-cell signaling, we devised a fluorescence micropipette assay to measure real-time T-cell calcium signaling at the single-cell level. Fast single T-cell calcium flux was observed upon T cell/APC contact (bond formation). Consistently, the calcium signaling amplitude and speed were dependent on the peptide potency (Fig. 5e, f, Fig. S15, and Movie S7). To link the TCR conformational change to TCR signaling, we simultaneously plotted the association of the TCR-CD3 Conformational change with the $\mathrm{Ca}^{2+}$ signal against the stimulation time. We found that the $\mathrm{CD} 3 \zeta$ conformational change was synchronized with the $\mathrm{Ca}^{2+}$ signal for all three different pMHCs (Fig. 5g). Together with the aforementioned smFRET measurements (Fig. 2), our data revealed that the TCR-pMHC bond conformation (Fig. 2d) governs the amplitude of $\mathrm{Ca}^{2+}$ release (Fig. 5e), possibly through bond distanceassociated mechanical forces. ${ }^{30,31} \mathrm{Ca}^{2+}$ release in turn drives the dissociation of positively charged $\mathrm{CD} 3 \zeta$ from the negatively charged phospholipids in the plasma membrane ${ }^{32}$ to expose the CD3 ITAM domains for phosphorylation via a $\mathrm{Ca}^{2+}$ signaling feedback loop (Fig. 5h).

According to the values for half-maximal T-cell proliferation $\left(E C_{50}\right)$ and the 3D half-life of pMHC tetramer binding reported by Corse et al., ${ }^{12}$ we then plotted the intermolecular TCR-pMHC conformations and the intramolecular TCR-CD3 3 distances against the values for the 2D on-rate, affinity, signaling, and proliferation and the 3D tetramer half-life (Fig. S16). Strong negative (Fig. 6a, solid dots and dashed lines) and positive (Fig. 6b, open dots and solid lines) correlations were found for the TCR-pMHC bond distances and the TCR-CD3 distances vs. all of the metrics of TCR binding, signaling, and activation, which govern the entire process of signal reception, transduction, and regulation, respectively. These measurements and correlations indicated the direct physiological relevance of the conformational dynamics of single TCR-pMHC bonds and individual TCR-CD3 complexes.

\section{DISCUSSION}

There is considerable controversy about TCR recognition and the initiation of signaling, which are key steps by which a TCR specifically and sensitively recognizes its agonist ligand and then transduces the recognition signal across the plasma membrane to cause alternations in the cytoplasmic portions of the associated CD3 signaling domains. To elucidate the molecular mechanisms underlying this critical process, we need to understand: (1) how the TCR discriminates between signals from a single agonist and those derived from the surrounding abundant self-peptides, and (2) how the TCR precisely propagates such a signal to CD3 to properly trigger T-cell activation. Here, we developed an in situ FRET method to measure the conformational changes of single TCR-pMHC bonds on the cell surface and individual TCR-CD3Z complexes across the cell membrane (Fig. 1a). Cell surface FRET1 revealed signal generation, and transmembrane FRET2 showed further signal propagation and amplification. The integration of both types of FRET could show the entire process of signal initiation for TCR triggering.

The TCR conformational change model has long been used to explain the mechanism underlying TCR triggering. However, neither crystal structures nor other biochemical assays have been able to provide solid evidence with enough spatiotemporal resolution to either prove or disprove this model. Here, we measured the intermolecular distances of single TCR-pMHC bonds using highly sensitive smFRET (Fig. 1a). In biochemistry, bond length, which is the average distance between the nuclei of two bonded atoms in a molecule, is used to describe the compactness of a bond. Because the TCR was labeled with a SCFv and CD4 was also bound to the PMHC, the distance measured here (Fig. 2d) did not reflect the bond length. However, the same scFv (Fig. 1a) was used for all three pMHCs, and CD4 binding contributed negligibly to the TCR-pMHC bond distances (Fig. 3b). The TCR-pMHC bond distances (Fig. 2d) measured in this study provided a reasonable approximation to determine the compactness (or bond length) of a TCR-pMHC bond, thus providing the most basic conformational information at the single-molecule level to advance the understanding of the molecular mechanism underlying TCR recognition in situ.

More importantly, the TCR-pMHC bond conformations measured by our smFRET assay were independent of the binding affinity and kinetics, although they were well correlated (Fig. 6). The smFRET assay is highly distance dependent and is only able to detect FRET signals when a TCR binds to a pMHC on the cell surface (Fig. 1b, d; comparison of K5 with null); ${ }^{8,33}$ in other words, smFRET only measured the conformational dynamics of single TCR-pMHC bonds. The bound state was confirmed by the presence of a nearly immobilized pMHC (diffusion coefficient $D_{\text {bound }} \approx 0$ ) for each smFRET trajectory, compared with the fast diffusion of free pMHCs (Fig. 2f, $g$ and Fig. S8). Such a dramatic diffusion difference has been previously used to differentiate bound and unbound pMHCs at the single-molecule level. ${ }^{19,20}$ By definition, the affinity is the ratio between bound and unbound molecules at the equilibrium phase, and kinetics describes the rates of bond association/dissociation. Because the TCR-pMHC bond conformation is not influenced by unbound molecules and bond association/dissociation, it is independent of the TCR-pMHC binding affinity and kinetics. In other words, the TCR-pMHC bond conformation measured by smFRET reveals an intrinsic property of the TCR/pMHC complex in the bound state.

Based on the observation that TCRs form microclusters during antigen recognition by conventional TIRF and superresolution microscopy, ${ }_{1}^{21,28,34}$ serial engagement has been proposed to explain the high sensitivity of T-cell recognition. ${ }^{2,3}$ To measure the conformational dynamics of single TCR-pMHC bonds without the possible influence of TCR clustering, we used the actin polymerization inhibitor LA to prevent the formation of TCR microclusters. Consistently, the smFRET measurements showed no differences in the TCR-pMHC bond distances measured in the presence or absence of TCR microclusters (Fig. 3c), which reinforced the importance of the single TCR-pMHC bond conformation in the triggering of T-cell signaling and revealed the high sensitivity of TCR recognition. ${ }^{1,2}$

Spatially, our data revealed strong correlations between the intermolecular TCR-pMHC conformation and the $\mathrm{Ca}^{2+}$ flux (Fig. 7a), the intramolecular TCR-CD3 3 distance (Fig. 7b), and CD3 3 phosphorylation (Fig. 7c). Our data showed that the strongest agonist, K5, formed the shortest TCR-pMHC bond, which caused the greatest dissociation of $C D 3 \zeta$ from the inner leaflet of the plasma membrane and lead to the maximum exposure of its ITAMs to result in the highest level of phosphorylation. In contrast, a structurally similar but weaker ligand, 102S, formed the longest bond with the TCR, resulting in the least dissociation of $\mathrm{CD} 3 \zeta$ from the membrane and the lowest level of phosphorylation. Our results collectively showed that a TCR discriminates between closely related peptides by forming TCR-pMHC bonds with different conformations, which precisely control the accessibility of CD3 ITAMs to phosphorylation (Fig. 7d). This discovery highlighted the critical importance of bond conformation in TCR triggering. Physiologically, our study suggested that a short bond, but not a long bond, can efficiently exclude the large, inhibitory tyrosine phosphatase CD45 to 


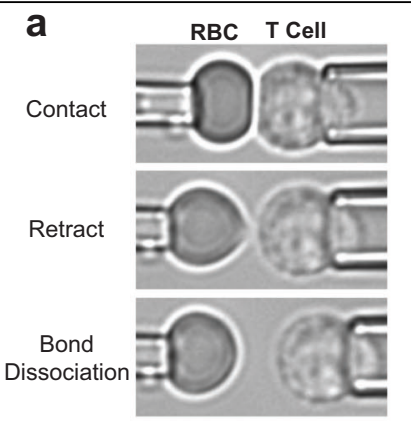

b

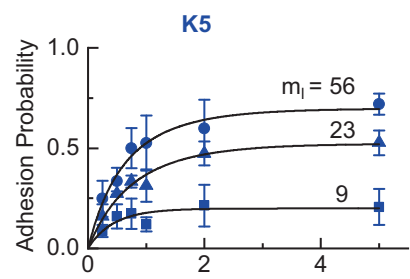

Contact Time (s)
C

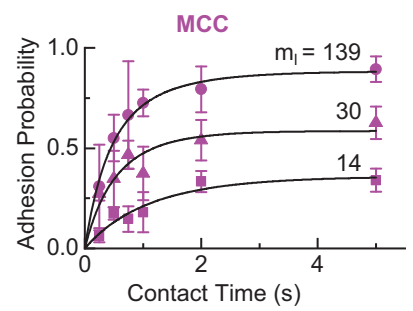

f

d e K5

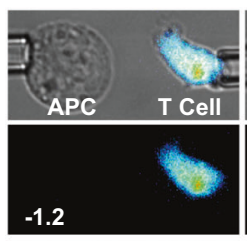

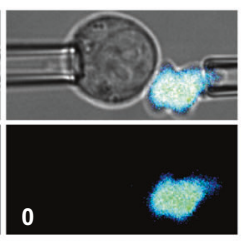
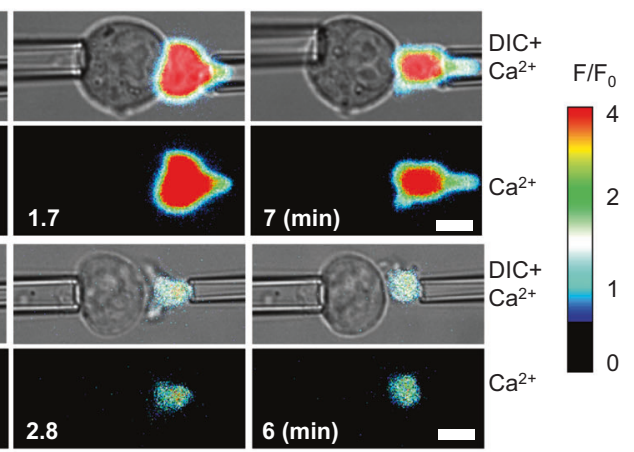

Null

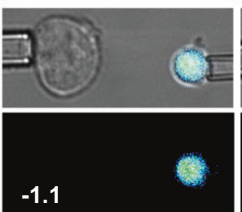

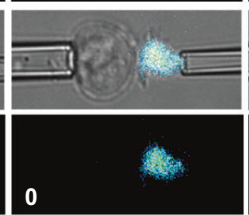

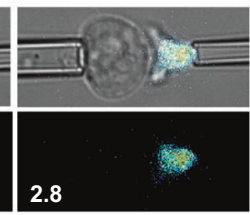

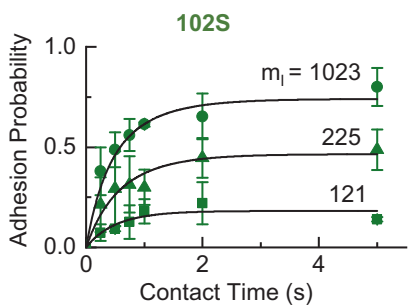

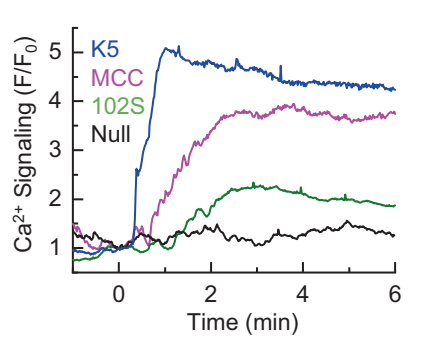

\section{g}
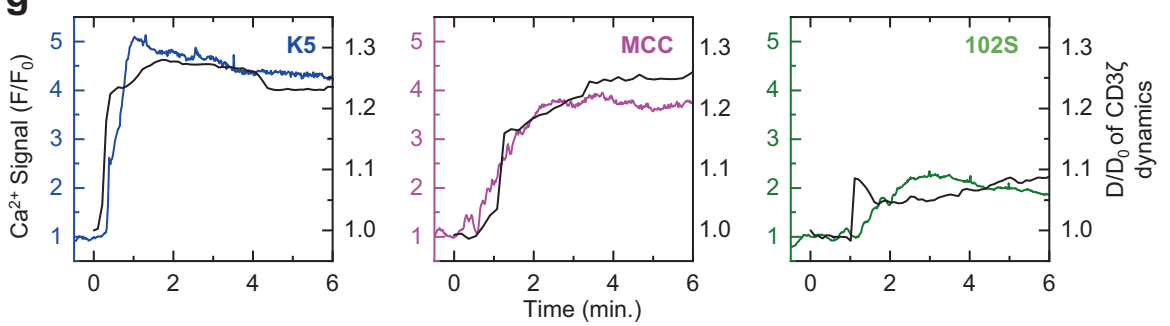

h

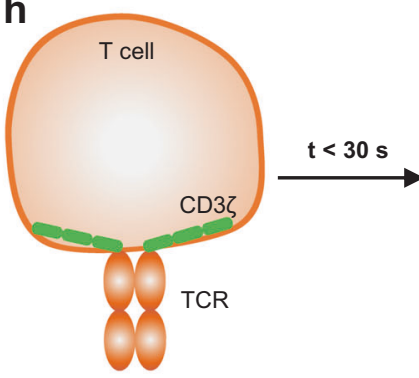

Resting state

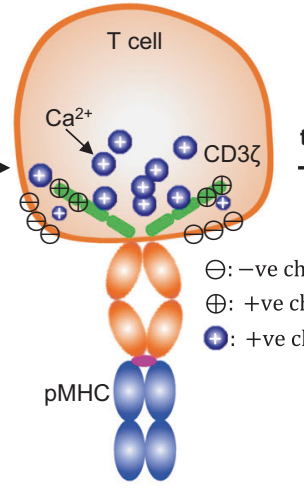

Initial CD3ろ dissociation and $\mathrm{Ca}^{2+}$ release

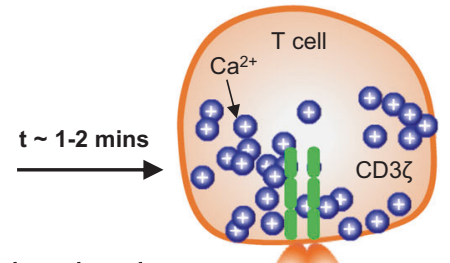

-ve charged membrane

: +ve charged CD3 3

: +ve charged $\mathrm{Ca}^{2+}$ ion

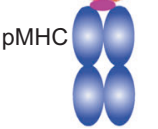

Amplified CD3 3

dissociation

Fig. $52 \mathrm{D}$ kinetics and T-cell $\mathrm{Ca}^{2+}$ signaling. a $2 \mathrm{D}$ micropipette adhesion frequency assay. A micropipette-aspirated T cell (right) was driven by a piezoelectric translator to make controlled contact with an RBC coated with pMHC held by another pipette (left). The retraction of T cells to the starting position resulted in elongation of the RBC, enabling the visual detection of the TCR-pMHC bond. Also see Fig. S15 and Movie S6. Adhesion curves for 5C.C7 TCRs interacting with the K5 (b), MCC (c), and $102 \mathrm{~S}$ (d) pMHCs measured by micropipette assay at $25^{\circ} \mathrm{C}$ at the indicated pMHC site densities. Each cell pair was tested 50 times with a given contact duration to estimate the adhesion probability, and three cell pairs were tested for each contact duration to calculate the mean adhesion probability. The data (points) were fitted by a probabilistic kinetic model (curves) to determine the 2D binding kinetics. The data are summarized in Table 1 and Table S1. e Real-time single T-cell calcium signaling measured by fluorescence micropipette. A CH27 cell loaded with the K5 (top row) or null peptide (bottom row, control) was precisely controlled to make contact with a primary $5 \mathrm{C} . \mathrm{C} 7 \mathrm{~T}$ cell loaded with the Fluo-4 calcium indicator at $37^{\circ} \mathrm{C}$. The fluorescence signal was recorded in real time by time-lapsed microscopy, and the fold increase in $\mathrm{Ca}^{2+}$ signaling $\left(F / F_{0}\right)$ is shown in pseudocolor. Representative $\mathrm{Ca}{ }^{2+}$ imaging experiments for the $\mathrm{K} 5$ and null peptides consisting of 6-8 independent experiments for each peptide are shown. Also see Fig. S15c, $d$ for the peptides MCC and 102S. See Movie S7 for more data. f Representative time trajectories for $\mathrm{Ca}^{2+}$ signaling stimulated by the K5, MCC, $102 \mathrm{~S}$, and null peptides. Fluorescence intensity values $(F)$ at any given timepoint were divided by the initial fluorescence intensity value at time zero $\left(F_{0}\right)$

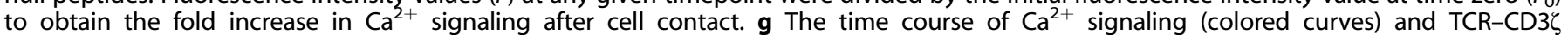
conformational changes (black curves). h TCR-CD3 $\zeta$ conformational changes were caused by a calcium signaling feedback loop. TCR-pMHC bond formation causes $\mathrm{CD} 3 \zeta$ dissociation and initiates $\mathrm{Ca}^{2+}$ flux, which in turn promotes $\mathrm{CD} 3 \zeta$ dissociation by neutralizing the negative charges of the anionic phospholipids in the T-cell membrane to fully expose the ITAMs on CD3 $\zeta$ to allow phosphorylation 
Table 1. 2D kinetic parameters

\begin{tabular}{|c|c|c|c|c|c|}
\hline Peptide & Sequence & T-cell activation & $A_{c} K_{a}\left(\mu m^{4}\right)$ & $k_{\text {off }}\left(\mathrm{s}^{-1}\right)$ & $A_{c} K_{\mathrm{on}}\left(\mu \mathrm{m}^{4} \mathrm{~s}^{-1}\right)$ \\
\hline MCC & ANERADLIAYLKQATK & Agonist & $1.5 \pm 0.7 \times 10^{-4}$ & $1.3 \pm 0.7$ & $2.0 \pm 1.1 \times 10^{-5}$ \\
\hline
\end{tabular}
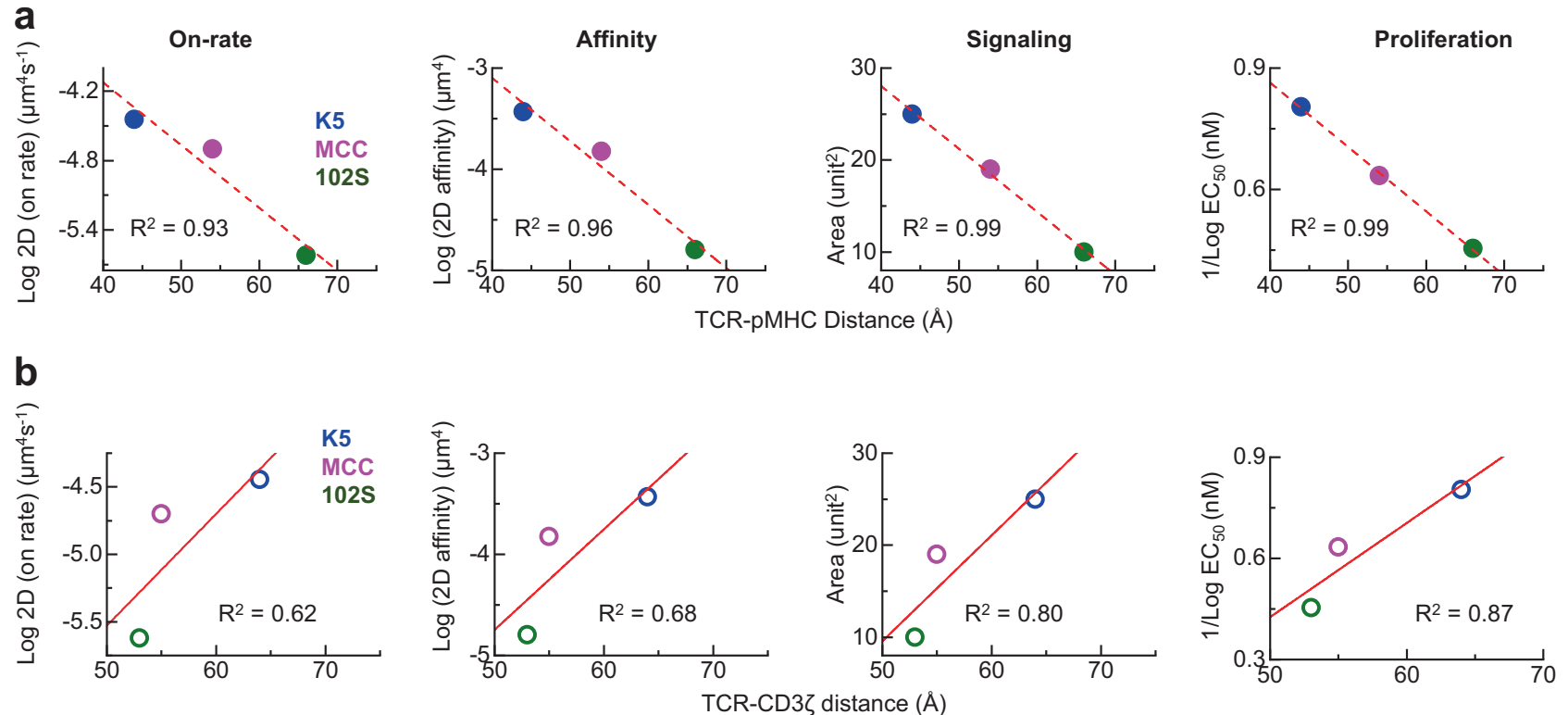

Fig. 6 Correlations between TCR conformations and T-cell binding kinetics, signaling, and proliferation. Correlations of the TCR-pMHC bond conformation (a) and TCR-CD3C distance (b) with the 2D on-rate, 2D affinity, calcium signaling, and proliferation for the K5, MCC, and 102S peptides. The $\mathrm{EC}_{50}$ data for cell proliferation were adapted from Corse et al. ${ }^{12}$ Data points were fitted with a linear function, and the goodness of correlation is indicated by the $R^{2}$ value

increase the phosphorylation of CD3 ITAMs by Lck. ${ }^{5,26}$ As bond conformation is independent of binding affinity and kinetics, the results of our study are also very well aligned with those of a previous study showing that elongating the $\mathrm{pMHC}$ ectodomain greatly reduces TCR triggering without affecting TCR-pMHC binding. ${ }^{17}$

Temporally, our results revealed that near-instantaneous TCR-pMHC binding (Fig. 2b) was concurrently followed by delayed $\mathrm{Ca}^{2+}$ flux and $\mathrm{CD} 3 \zeta$ disassociation (Fig. $5 \mathrm{~g}$ ), which was in line with the observation in a previous work that $\mathrm{Ca}^{2+}$ and $\mathrm{CD} 3 \varepsilon / \zeta$ phosphorylation use a positive feedback loop to amplify and sustain T-cell signaling. ${ }^{32}$ Together with the results of previous studies, our data suggested a "TCR-pMHC bond conformational change model" (Fig. 7d) in which a TCR deciphers the structural differences between foreign and self-peptides by forming TCR-pMHC bonds with different distances, which most likely trigger an increase in $\mathrm{Ca}^{2+}$ signaling that is proportional to the bond distance through bond distance-controlled mechanical forces; ${ }^{3,30,31,35}$ a previous study has shown that mechanical forces are required to trigger $\mathrm{Ca}^{2+}$ flux. ${ }^{30}$ In turn, the released $\mathrm{Ca}^{2+}$ regulates the dissociation of positively charged $\mathrm{CD} 3 \zeta$ cytoplasmic domains from negatively charged phospholipids in the plasma membrane (Fig. $5 \mathrm{~h}$ ). Such a $\mathrm{Ca}^{2+}$ positive signaling feedback loop propagates and amplifies the differences between foreign and self-peptides until TCR-pMHC bond dissociation occurs. ${ }^{36}$ This bond conformational change model (Fig. 7d) is compatible with existing TCR triggering models $s^{5,6}$ and explains the high specificity and sensitivity of T-cell recognition and the results of previous studies of CD3 conformational changes. ${ }^{9,37-40}$ Our model suggests that $T$ cells use accurate, reliable, and efficient machinery to faithfully transduce extracellular TCR-pMHC binding into appropriate intracellular signals to ensure the optimal spatial and temporal activation of $\mathrm{T}$ cells.

In summary, our study revealed the dynamic process underlying the manner in which TCR recognition signals are initiated, controlled, transmitted, and amplified via the direct linking of the intermolecular TCR-pMHC conformation and the intramolecular TCR-CD3 , distance to T-cell surface binding, intracellular signaling, and functional responses. This sheds light on the molecular mechanisms by which a TCR deciphers the structural differences between foreign and self-peptides via the TCR-pMHC bond conformation to initiate and amplify different TCR signaling responses for ligand discrimination.

\section{EXPERIMENTAL PROCEDURES}

Mice

The Institutional Animal Care and Use Committee of the University of Chicago approved the animal protocols used in this study. The 5C.C7 TCR transgenic RAG2 knockout mice with a B10.A background were a generous gift from the NIAID.

Cells

T-cell blasts were obtained by stimulating splenocytes isolated from 5C.C7 TCR transgenic mice with $10 \mu \mathrm{M}$ MCC peptide (amino acids 88-103, ANERADLIAYLKQATK) according to a protocol approved by the Institutional Animal Care and Use Committee of the University of Chicago. The T-cell blasts were maintained in complete medium 
a

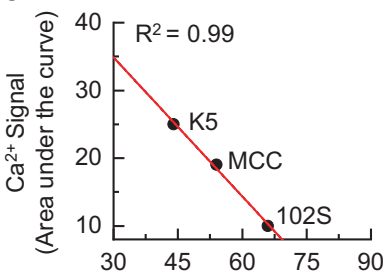

d

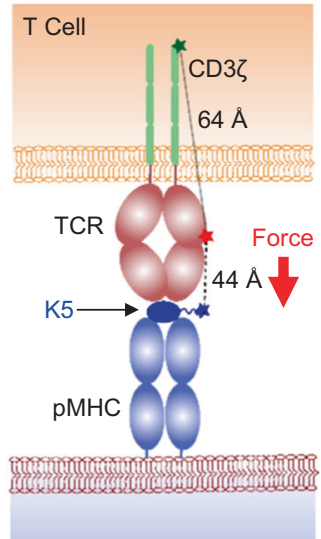

b
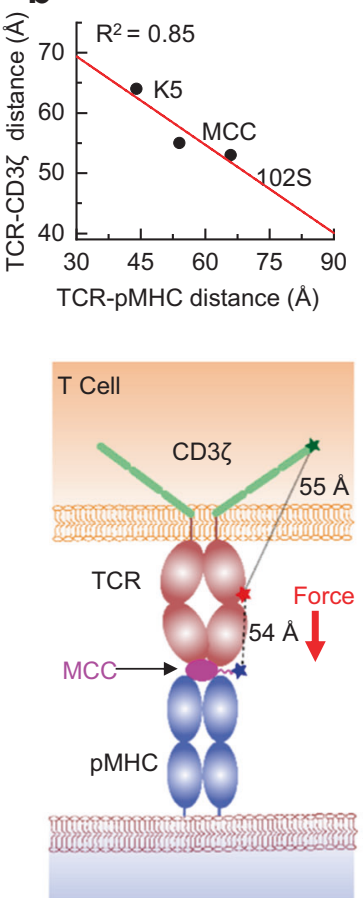
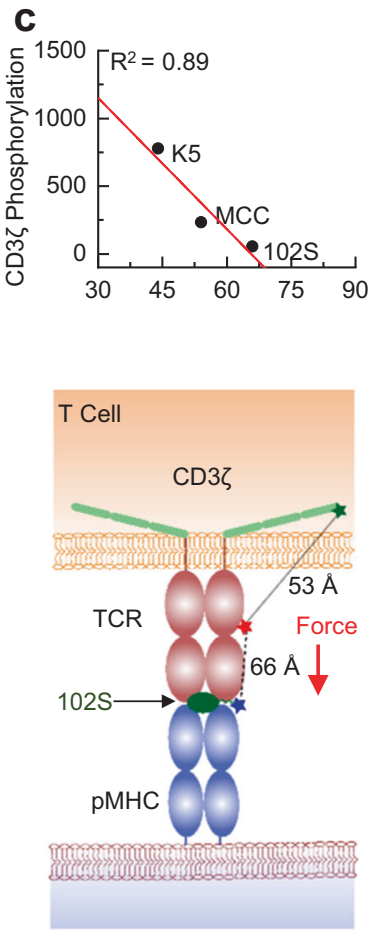

Fig. 7 Mechanistic models of TCR triggering and discrimination. Correlations between TCR-pMHC bond conformation and calcium flux (a),

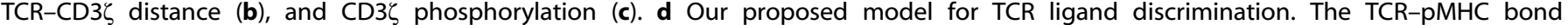
conformation controls the TCR-CD3 $\zeta$ distance to regulate the exposure of ITAMs on CD3 $\zeta$ for subsequent phosphorylation for the K5, MCC, and $102 \mathrm{~S}$ pMHCs, respectively

(RPMI 1640 medium with 10\% fetal bovine serum (FBS), 2 mM Lglutamine, $50 \mu \mathrm{M} \beta$-mercaptoethanol, and penicillin-streptomycin). The T cells were used on days 6-9 for imaging and the micropipette experiments. The live $T$ cells were separated from the dead cells by Ficoll-Paque density gradient media (GE). B-cell lymphoma $\mathrm{CH} 27$ cells were used as APCs. The APCs were maintained in the same medium that was used for the T cells. ${ }^{2}$ Human RBCs were isolated from the whole blood of healthy donors from the hospital at the University of Chicago according to a protocol approved by the Institutional Review Board of the University of Chicago.

\section{Reagents}

1-palmitoyl-2-oleoyl-sn-glycero-3-phosphocholine (POPC), 1,2-dioleoyl-sn-glycero-3-[(N-(5-amino-1-carboxypentyl) iminodiacetic acid) succinyl] nickel salt (DGS-NTA-Ni ${ }^{2+}$ ), and 1,2-dioleoyl-sn-glycero-3phosphoethanolamine-N [methoxy (polyethyleneglycol)-5000] ammonium salt (PEG5000 PE) were purchased from Avanti Polar Lipids. PBS, BSA, FBS, and Alexa Fluor 568 C5 maleimide were purchased from Thermo Fisher Scientific. PEG-NTA-Ni ${ }^{2+}$-coated coverslips were purchased from MicroSurfaces, Inc. Nunc Lab-Tek eight-well chambered coverslips were purchased from Thermo Fisher Scientific. His-tagged B7 was generated as previously reported. ${ }^{8}$ His-tagged ICAM-1 was purchased from Sino Biological. Cy3 and Cy 5 maleimide mono-reactive dyes were purchased from GE Life Sciences. Alexa Fluor 568, Fura-4, and di-methyl sulfoxide were purchased from Thermo Scientific. LB media was obtained from Fisher Scientific.

\section{pMHCs}

For the FRET measurements between TCRs and pMHCs on the cell surface (FRET1), we generated a peptide-exchangeable CLIP-IE ${ }^{k}$ molecule. ${ }^{41}$ The $a$ and $\beta$ chains of the CLIP-IE ${ }^{\mathrm{K}}$ molecule were cloned into pAC vectors with a gp67 signal sequence (BD Biosciences), acidic or basic leucine zipper sequences and a 6-histidine tag at the C-terminus. Primary baculoviruses were prepared for each chain by cotransfecting the construct with linearized baculovirus DNA (BestBac 2.0, Expression Systems) into Sf9 cells using CellFectin reagent (Thermo Fisher Scientific). The cells were washed and incubated at $27^{\circ} \mathrm{C}$ for 1 week. The primary viruses were harvested by centrifugation and the collection of the supernatant. Baculoviruses were amplified to generate higher titers by infecting Sf9 cells for another week. Hi5 cells were coinfected with baculoviruses encoding both chains, and the supernatants were harvested after $65 \mathrm{~h}$ of infection. The $\mathrm{pH}$ of the supernatant was adjusted to $\mathrm{pH} 6.9$ with HEPES-buffered saline (10 mM HEPES pH 7.2, $150 \mathrm{mM} \mathrm{NaCl}$, and $\left.0.02 \% \mathrm{NaN}_{3}\right), 20$ $\mathrm{mM}$ imidazole, $\mathrm{pH} 7.2,5 \mathrm{mM} \mathrm{MgCl}$, and $0.5 \mathrm{mM} \mathrm{NiCl}_{2}$. Ni-NTA agarose (Qiagen) was added to the supernatant and stirred overnight at $4{ }^{\circ} \mathrm{C}$. The supernatant was filtered, the Ni-NTA agarose was collected, and CLIP-IE ${ }^{\mathrm{K}}$ was eluted using $200 \mathrm{mM}$ imidazole, $\mathrm{pH} 7.2$, in HBS. The protein fractions were analyzed by SDS-PAGE. CLIP-IE ${ }^{\mathrm{K}}$ was purified using Superdex 200 size-exclusion column chromatography and Mono-Q anion exchange chromatography (GE healthcare). The purified fractions were used for peptide loading. According to a method described in a previous publication, ${ }^{8}$ peptides with fluorescent maleimide dye (Cy3) at the C-terminus, including K5(C)-ANERADLIAYFKAATKFGGdSdC, MCC(C)-ANERADLIAYLKQATKGGdSdC, T102S(C)-ANERADLIAYLKQASKGGdSdC, and null(C)-ANERAELIAYLTQAAKGGdSdC, were synthesized, labeled with Cy3, purified by HPLC, and verified by mass spectrometry by the Elim Biopharm Company (CA). The peptides of interest were added to the purified CLIP-IE ${ }^{k}$ protein at a 100-fold molar excess for the peptide exchange reaction. Thrombin $\left(1 \mathrm{U} / 100 \mu \mathrm{g}\right.$ of $\left.I \mathrm{E}^{\mathrm{k}}\right)$ was added and incubated at $37^{\circ} \mathrm{C}$ for $1 \mathrm{~h}$. The $\mathrm{pH}$ of the solution was decreased by adding MES buffer, $\mathrm{pH} 6.2$, at a final concentration of $30 \mathrm{mM}$, and the $\mathrm{IE}^{\mathrm{K}}$ was again incubated at $37{ }^{\circ} \mathrm{C}$ overnight. The $\mathrm{pH}$ of the protein solution was adjusted with $40 \mathrm{mM}$ HEPES, pH 7.2. Extra peptides and denatured proteins were removed by centrifugation $(16,000 \times g$ for $30 \mathrm{~min}$ at 
$4^{\circ} \mathrm{C}$ ) and desalting twice with Zeba Spin Desalting Columns (Thermo Fisher).

For the FRET measurements between TCR and CD3 3 across the cell membrane (FRET2) and the 2D micropipette adhesion assays, $\mathrm{IE}^{\mathrm{k}}$ pMHC monomers generated by the NIH Tetramer Core Facility were used. Biotinylated monomeric $\mathrm{IE}^{\mathrm{k}}$ was covalently complexed with the ANERADLIAYFKAATKF (K5), ANERADLIAYLKQATK (MCC), ANERADLIAYLKQASK (102S), and PVSKMRMATPLLMQA (human CLIP 87-101) peptides. The $I^{\mathrm{k}}$ monomers were aliquoted and stored at $-80^{\circ} \mathrm{C}$ until use.

\section{Production and labeling of the scFvs}

Plasmid constructs encoding two mutants of the anti-TCR scFvs $\mathrm{J} 1$ and $\mathrm{J} 3$ were obtained as a generous gift from Mark M. Davis at Stanford University. ${ }^{8} \mathrm{~J} 1$ was used for cell surface FRET1, and J3 was used for the transmembrane FRET2 experiments. To generate the $\mathrm{J} 1$ and $\mathrm{J} 3$ proteins, BL21 bacteria were transfected with CDNA and cultured on a large scale ( $2 \mathrm{~L}$ each) in the presence of isopropyl $\beta$-D-1-thiogalactopyranoside. The bacteria were spun down, and the cell pellets were resuspended with bacterial-protein extraction reagent (Thermo Fisher), lysozyme, and DNase, followed by washing with inclusion wash buffer ( $100 \mathrm{mM} \mathrm{NaCl}, 50 \mathrm{mM}$ Tris base, and $0.05 \%$ (volume) Triton X$100) .{ }^{42}$ The refolding and purification of the recombinant scFvs were performed using a modified method based on previous publications. $^{43,44}$ In brief, the scFvs were unfolded in the presence of $10 \mathrm{mM} \beta$-mercaptoethanol for $2 \mathrm{~h}$ at $25^{\circ} \mathrm{C}$ (in $100 \mathrm{~mL}$ of $100 \mathrm{mM}$ Tris- $\mathrm{HCl}$ buffer with $\mathrm{pH}=8.0,6 \mathrm{M} \mathrm{GuHCl}$ and $200 \mathrm{mM} \mathrm{NaCl}$ ), so that they could be refolded to obtain proteins with the correct conformations by stepwise dialysis methods without causing protein oxidation. To remove the reducing agent, the denatured recombinant scFvs were dialyzed against $1 \mathrm{~L}$ of $100 \mathrm{mM}$ Tris- $\mathrm{HCl}, \mathrm{pH} 8.0$, buffer with $6 \mathrm{M} \mathrm{GuHCl}$ and 200 $\mathrm{mM} \mathrm{NaCl}$ for $15 \mathrm{~h}$ at $4{ }^{\circ} \mathrm{C}$ with gentle stirring. Then, stepwise dialyses were performed in the same Tris- $\mathrm{HCl}$ buffer containing decreasing concentrations of $\mathrm{GuHCl}(4,2,1,0.5$, and $0 \mathrm{M})$ for 15 $\mathrm{h}$ for each step at $4{ }^{\circ} \mathrm{C}$ with gentle stirring. During the 1 and 0.5 $\mathrm{M}$ dialysis steps, $400 \mathrm{mM}$ L-arginine (Sigma-Aldrich) and $375 \mu \mathrm{M}$ of oxidized glutathione (Sigma-Aldrich) were added. The final dialysis was performed in buffer without $\mathrm{GuHCl}$ for $18 \mathrm{~h}$ at $4{ }^{\circ} \mathrm{C}$ with gentle stirring. The protein was concentrated and stored at $4{ }^{\circ} \mathrm{C}$ prior to long-term storage at $-20^{\circ} \mathrm{C}$ in the presence of $50 \%$ glycerol. The purified scFVs were labeled with Cy5-malimide in the presence of $50 \mu \mathrm{M}$ Tris-(2-carboxyethyl) phosphine hydrochloride for $2 \mathrm{~h}$ at room temperature followed by $12 \mathrm{~h}$ of gentle mixing at $4{ }^{\circ} \mathrm{C}$. Then, the labeled scFVs were purified by a resin spin column. J1 and J3 were labeled and purified for the FRET experiments. The binding specificity of each of the scFvs for the 5C.C7 TCRs was confirmed by flow cytometry before the imaging experiments.

\section{CD3Z-GFP transduction}

Primary 5C.C7 T cells were retrovirally transduced with CD3Z-GFP according to a previously published method. ${ }^{45}$ Ecotropic platinum-E retroviral packaging cells were transiently transfected with the MIG-CD3Z-GFP vector by calcium phosphate precipitation. The viral supernatant was harvested twice at 48 and $72 \mathrm{~h}$ post transfection, filtered by a $0.2 \mu \mathrm{m}$ cellulose acetate membrane, and used for the subsequent experiments. Splenocytes isolated from 5C.C7 mice were cultured in RPMI supplemented with $10 \%$ FBS, $2 \mathrm{mM}$ glutamine, $50 \mu \mathrm{M}$ 2-mercaptoethanol, $1 \mathrm{mM}$ HEPES, 1 $\mathrm{mM}$ sodium pyruvate, $1 \times$ glutamine and nonessential amino acids (Thermo Fisher), $100 \mathrm{U} / \mathrm{mL}$ penicillin, $100 \mu \mathrm{g} / \mathrm{mL}$ streptomycin, and $50 \mu \mathrm{g} / \mathrm{mL}$ gentamycin and stimulated with precoated 1.5 $\mu \mathrm{g} / \mathrm{mL}$ anti-CD3E Ab (Clone 145-2C11, University of Chicago Monoclonal Antibody Facility) and $0.5 \mu \mathrm{g} / \mathrm{mL}$ anti-CD28 Ab (Clone 37.51 , Biolegend) in the presence of $40 \mathrm{U} / \mathrm{mL}$ recombinant human IL-2 (Peprotech). After $24 \mathrm{~h}$ of cell activation, $2 \mathrm{~mL}$ viral supernatant was added to a well of a Retronectin (Clontech)precoated $\left(12.5 \mu \mathrm{g} / \mathrm{mL}\right.$ in PBS, incubated at $4{ }^{\circ} \mathrm{C}$ overnight) six-well plate and centrifuged for $90 \mathrm{~min}$ at $3000 \times \mathrm{g}$, and then the stimulated cells were transferred to the plate and centrifuged in the viral supernatant supplemented with $4 \mu \mathrm{g} / \mathrm{mL}$ protamine sulfate at $800 \times g$ for $90 \mathrm{~min}$. The transduction rate of $\mathrm{CD} 3 \zeta-\mathrm{GFP}$ was determined by GFP fluorescence at least $16 \mathrm{~h}$ after transduction.

\section{Lipid bilayer preparation}

The glass-supported lipid bilayer preparation method was developed based on previous publications. ${ }^{25,46}$ The lipid layer was generated by mixing POPC (90\%), DGS-NTA-Ni ${ }^{2+}(9.9 \%)$, and PEG500PE $(0.1 \%)$ in chloroform in clean glass vials. The chloroform was dried with $0.22-\mu \mathrm{m}$-filtered $\mathrm{N}_{2}$, and then vials containing the lipid layer were kept in a vacuum for $2 \mathrm{~h}$ to dry them completely. The lipid layer was then resuspended in filtered PBS buffer $(\mathrm{pH} \mathrm{7.4;}$ Clontech) at a concentration of $4 \mu \mathrm{M} / \mathrm{mL}$. To decrease the sizes of the multilamellar lipid vesicles to generate unilamellar vesicles, the cloudy vesicle solution was repeatedly frozen in liquid nitrogen and thawed in a $37^{\circ} \mathrm{C}$ water bath 30 times until the solution became clear. The unilamellar vesicle solution was stored at $-80^{\circ} \mathrm{C}$ for future experiments. Before each experiment, a tube was centrifuged at $33,000 \times g$ for $45 \mathrm{~min}$ at $4^{\circ} \mathrm{C}$. The supernatant was incubated for $90 \mathrm{~min}$ on an eight-well Lab-Tek chamber coverslip that was thoroughly prewashed twice with $5 \mathrm{~N} \mathrm{NaOH}$ at $50^{\circ} \mathrm{C}$, followed by washing with PBS twice at $37^{\circ} \mathrm{C}$. The lipid vesicles fused onto the glass surface and formed a glasssupported lipid bilayer. The lipid bilayer was washed three times thoroughly with PBS to remove excess lipids. Then, a mixture of His-tagged, fluorescently labeled pMHCs and His-tagged, nonfluorescent ICAM-1, and B7 was added to the lipid bilayer and incubated for $1 \mathrm{~h}$. After $30 \mathrm{~min}$, the unbound proteins were washed with PBS three times. The bound protein was incubated for another $30 \mathrm{~min}$ at $37^{\circ} \mathrm{C}$, and the weakly bound proteins were washed away with PBS three additional times. The protein-bound lipid was incubated with $1 \%$ BSA for 20 min to minimize the background fluorescence during microscopy experiments. The excess BSA was washed away three times with PBS. The microscopy experiments were performed using imaging buffer (PBS, pH 7.4, $137 \mathrm{mM} \mathrm{NaCl}, 5 \mathrm{mM} \mathrm{KCl}, 1 \mathrm{mM} \mathrm{CaCl} 2,2 \mathrm{mM}$ $\mathrm{MgCl}_{2}, 0.7 \mathrm{mM} \mathrm{Na}_{2} \mathrm{HPO}_{4}, 6 \mathrm{mM} \mathrm{D}$-glucose, and 1\% BSA).

The fluidity and integrity of the lipid bilayer were tested by fluorescence recovery after photobleaching experiments with 32 nM Cy3-labeled pMHC reconstituted on a supportive lipid bilayer (Fig. S2a and Movie S2). The photobleaching was performed by using a high power $(60 \mathrm{~mW}) 532-\mathrm{nm}$ CW laser with $5 \mathrm{~s}$ of exposure at the center of the imaging area, and the fluorescence recovery of the lipid bilayer was imaged with a $470 \pm 10 \mathrm{~nm}$ LED light at $10 \mathrm{~s}$ intervals. The power and duration of the laser and LED light excitation were controlled by analog modulation. The diffusion coefficient $(D)$ was determined by labeling the lipid bilayer with $1 \mathrm{nM}$ of Cy3-labeled pMHCs, which were excited by a stable 532-nm laser (Fig. S2g). The diffusion of the single pMHCs in the lipid bilayer was tracked in real time and characterized (Fig. S2b-f and Movie S1). The experimentally determined diffusion coefficient $D_{e}$ was verified by a small-scale simulation $D_{s}$, which showed high similarity (Fig. S2h-k). The pMHC diffusion constant was determined by the TrackArt program in MATLAB and was consistent with previously reported values. ${ }^{47}$

\section{Microscopy}

All imaging experiments were performed using our custom-built TIRF and epifluorescence microscope setup, which utilized a NikonTi-E inverted microscope attached to an Optosplit III (CAIRN Research) and an Andor iXon 888 EMCCD camera $(1024 \times 1024$ pixel) (Fig. S1b). The individual of 405, 488, 532, and 647-nm (Cobolt) CW laser lines were aligned to an achromatic fiber port 
(APC type, 400-700 nm, Thorlabs, Inc.) and then passed through an achromatic polarization-maintaining single-mode fiber to a motorized Nikon TIRF illuminator. The lasers were directed by a custombuilt quad-band dichroic mirror (Chroma, ZT405-488-532-640rcp) to the sample through a 1.42 NA $100 \times$ TIRF objective. A sevencolor solid-state LED light source with bandpass filters was also attached with a liquid light guide to the upper filter cube wheel in the Nikon microscope. The fluorescence from the donor and acceptor passed through a quad-band laser clean-up filter (ZET405$488-532-647 \mathrm{~m}$ ) and then passed through the Optosplit III to separate the emission fluorescence of the FRET donor and the acceptor. In the Optosplit cube, we used different filter sets for the FRET1 and FRET2 experiments. For FRET1 (Cy3-Cy5), we used T640lpxr-UF2 (Chroma) as a dichroic filter, ET585/65 for the Cy3 channel (Chroma), and ET655lp for the Cy5 channel (Chroma) for the individual fluorescence signals. For FRET2 (GFP-Alexa568), we used the dichroic filter T560lpxr-UF2 and two bandpass filters: Chroma ET510/20m for GFP and Chroma ET595/50m for Alexa568. For single-molecule imaging, we used hardware sequencing to obtain the highest frame rate (5-10 ms exposure) using high EM gain. Either hardware sequencing (via the camera) or software sequencing (Micromanager) was utilized with analog modulation to synchronize the image acquisition by the EMCCD camera, which triggered each laser and individual LED source. The donor and acceptor signal channels were physically separated by the beam splitter in the Optosplit III, and both signals were imaged simultaneously on the same image frame. The hardware stage control information and images were acquired by Micromanager. ${ }^{48}$

\section{FRET experiments}

For the intermolecular TCR-pMHC FRET1 measurements, T cells were incubated with a saturated concentration $(0.027 \mu \mathrm{g} / \mu \mathrm{L}$, Fig. S17a) of Cy5-labeled scFv J1 for $30 \mathrm{~min}$ at $4{ }^{\circ} \mathrm{C}$. After three washes, the cells were added to a lipid bilayer containing Cy3labeled pMHCs $\left(0.3\right.$ molecules $\left./ \mu \mathrm{m}^{2}\right)$ to perform smFRET using TIRF microscopy in a stream model. To perform the bulk FRET1 experiments, we used a pMHC density of 95 molecules $/ \mu \mathrm{m}^{2}$ in the lipid bilayer. For the intramolecular TCR-CD3 FRET2 measurements, $T$ cells transduced with CD3Z-GFP were labeled with a saturated concentration $(0.027 \mu \mathrm{g} / \mu \mathrm{L}$, Fig. S17a) of Alexa568-labeled scFv J3 for $30 \mathrm{~min}$ at $4{ }^{\circ} \mathrm{C}$. After three washes, the cells were added to a lipid bilayer containing unlabeled pMHCs to perform transmembrane FRET2 using epifluorescence microscopy.

Density quantification of the TCRs and pMHCs

The average surface TCR density in the 5C.C7 T cells (labeled with a saturated concentration of $\mathrm{H} 57 \mathrm{scFv}$ ) in the contact area between a $T$ cell and the lipid bilayer were quantified based on the fluorescence signal of a single Cy5- or Alexa568-labeled $\mathrm{H} 57 \mathrm{scFv}$. The molecular density of pMHCs in the lipid bilayer was estimated by dividing the total number of pMHC molecules by the total surface area as described previously. ${ }^{25}$

\section{CD4 blockade and pharmacological treatments}

To measure the TCR-pMHC bond distance by smFRET in the absence of CD4 binding, T cells were incubated with $10 \mu \mathrm{g} / \mathrm{mL}$ anti-CD4 antibody (GK 1.5, Biolegend) and $0.027 \mu \mathrm{g} / \mu \mathrm{L}$ of Cy5-J1 scFV for $30 \mathrm{~min}$ at $4{ }^{\circ} \mathrm{C}$. After three washes, the cells were added to a lipid bilayer containing Cy3-pMHCs, and smFRET assays were performed in the continuous presence of $10 \mu \mathrm{g} / \mathrm{mL}$ anti-CD4 antibody at $37^{\circ} \mathrm{C}$ using TIRF microscopy. To measure the TCR-pMHC bond distance by smFRET without the formation of TCR microclusters, T cells were incubated with $1 \mu \mathrm{M}$ LA (SigmaAldrich) and $0.027 \mu \mathrm{g} / \mu \mathrm{L}$ of Cy5-J1 scFv for $1 \mathrm{~h}$ at $25^{\circ} \mathrm{C}$. The cells were washed three times, and SmFRET assays were performed with a lipid bilayer containing Cy3-labeled pMHCs in the continuous presence of $1 \mu \mathrm{M} \mathrm{LA}{ }^{3}$ at $37^{\circ} \mathrm{C}$ using TIRF microscopy.
To measure the TCR-CD3 $\zeta$ conformational dynamics in the absence of TCR signaling, T cells were incubated with $10 \mu \mathrm{M}$ PP2 (Sigma-Aldrich) and $0.027 \mu \mathrm{g} / \mu \mathrm{L}$ of Cy5-J1 scFv for $1 \mathrm{~h}$ at $25^{\circ} \mathrm{C}$. The cells were washed three times, and smFRET assays were performed with a lipid bilayer containing Cy3-labeled pMHCs in the continuous presence of $10 \mu \mathrm{M} P P 2^{49}$ at $37^{\circ} \mathrm{C}$ using epifluorescence microscopy. In parallel, to demonstrate the role of PP2 in blocking TCR signaling, CD3ろ-GFP T cells were loaded with a calcium indicator dye (X-Rhod-5F, Thermo Fisher Scientific), and the real-time calcium flux was measured at $37^{\circ} \mathrm{C}$ using epifluorescence microscopy.

\section{Anisotropy measurements}

Anisotropy measurements (Fig. S17b) were performed as previously described. ${ }^{50}$ Briefly, a polarized $532-\mathrm{nm}$ laser was used to excite the samples, and the emission was split by a Wollaston prism (CAIRN Res.) into parallel and perpendicular polarized components that were imaged simultaneously with an EMCCD. The fluorescence anisotropy was determined by the following equation: ${ }^{51}$

$r(t)=I_{\|}-G I_{\perp} / I_{\|}+2 G I_{\perp}$,

where $I_{\|}$and $I_{\perp}$ are the fluorescence intensities of the parallel $(\|)$ and perpendicular $(\perp)$ polarized emission components with respect to the vertically polarized excitation. The $G$-factors were calculated according to previously reported methods. ${ }^{51}$

\section{D fluorescent micropipette assays}

The micropipette apparatuses were constructed by using a Leica inverted microscope placed on an anti-vibration table (Newport) equipped with manometer systems to apply suction pressure through glass pipettes (Fig. S15a). Two opposing pipettes were attached to two identical piezoelectric micromanipulators (Sensapex) to control the contacts between a T cell and a pMHC-coated RBC or $\mathrm{CH} 27$ APC. In the micropipette apparatus, one of the pipettes was also attached to a PI piezo actuator to allow computer-controlled fine movements for the repeated adhesion test cycles. A cell chamber of the desired size was prepared by cutting a coverslip. The temperature of the cell chamber $\left(37^{\circ} \mathrm{C}\right)$ was controlled by an objective heater (Bioptechs). To avoid medium evaporation during heating, the chamber was sealed with mineral oil (Sigma). The real-time images were acquired by an Andor iXon 888 EMCCD camera with a 100x objective and Micromanager software. For real-time calcium imaging, the sample was illuminated by sequentially triggered exposure to $470 \pm 20 \mathrm{~nm}$ blue light (Spectra X, Lumencor) and white LED light. The triggering of the light channels and the data acquisition were performed with analog modulation using Micromanager. ${ }^{48}$ For the 2D kinetic measurements, only continuous white LED light was used to detect the adhesion between a $\mathrm{T}$ cell and a pMHCcoated RBC.

\section{D micropipette kinetic assays}

The 2D micropipette adhesion experiments 3 were performed using T-cell blasts ${ }^{2}$ and pMHC-coated RBCs. Monomeric pMHCs were coated onto RBCs by biotin-streptavidin coupling. RBCs isolated from whole blood were biotinylated using different concentrations of biotin-X-NHS according to the manufacturer's instructions. Ten microliters of RBC solution $\left(\sim 10 \times 10^{6}\right)$ with $2 \mathrm{mg} / \mathrm{mL}$ streptavidin solution $(10 \mu \mathrm{l})$ were mixed for $30 \mathrm{~min}$ at $4{ }^{\circ} \mathrm{C}$ and then incubated with $20 \mu \mathrm{g} / \mathrm{mL}$ biotinylated pMHC monomer for $30 \mathrm{~min}$ at $4{ }^{\circ} \mathrm{C}$. After each step, the RBCs were washed three times.

To determine the surface TCR and PMHC densities, T cells were incubated with $10 \mu \mathrm{g} / \mathrm{mL}$ of the PE-conjugated anti-mouse TCR $\beta$ chain antibody clone H57-597 (BD) in $200 \mu \mathrm{L}$ of FACS buffer (RPMI $1640,5 \mathrm{mM}$ EDTA, $1 \% \mathrm{BSA}$, and $0.02 \%$ sodium azide) at $4{ }^{\circ} \mathrm{C}$ for $30 \mathrm{~min}$, and the PMHC-coated RBCs were stained with the PE- 
conjugated anti-IE ${ }^{k}$ clone 14.4.4s (BD). The T cells and RBCs were analyzed by a BD LSRFortessa flow cytometer. The fluorescence intensities were compared with those of standard calibration beads (BD Quantibrite PE Beads, BD) to determine the total number of molecules per cell, which were divided by the cell or bead surface area to obtain the site densities. The apparent surface area of a T cell $\left(523 \mu^{2}\right)$ and an RBC $\left(140 \mu \mathrm{m}^{2}\right)$ were calculated according to the areas of smooth spheres based on the microscopic measurement of their radii.

T cells were incubated at $37^{\circ} \mathrm{C}$ with a saturating concentration $(10 \mu \mathrm{g} / \mathrm{mL}$ ) of purified anti-mouse CD4 (clone GK 1.5, BD) prior to their addition to the chamber. The RBC was moved in and out of contact with the T cell to maintain specific contact times $(0.25,0.5$, $0.75,1,2$, and $5 \mathrm{~s}$ ) and area by a computer program. The adhesion events were detected by observing RBC elongation upon cell separation. The contact-retraction cycle was repeated 50 times for each given contact time. The specific adhesion probability $\left(P_{a}\right)$ for each contact timepoint was calculated by subtracting the nonspecific adhesion frequency $\left(P_{\text {nonspecific }}\right)$. The following equations were used to analyze the data.

$P_{a}$ vs. contact time $t$ were fitted using a probabilistic model (Eq. 1): ${ }^{52}$

$P_{a}=1-\exp \left\{-m_{r} m_{l} A_{c} K_{a}\left[1-\exp \left(-k_{r} t\right)\right]\right\}$,

where $K_{a}$ and $k_{r}$ are the 2D binding affinity and off-rate, $m_{r}$ and $m_{l}$ are the respective TCR and pMHC densities that were measured by flow cytometry, and $A_{c}$ is the contact area. The curve fitting generates two parameters, the effective $2 \mathrm{D}$ affinity $A_{c} K_{a}$ and the 2D off-rate $k_{r}$. Its product with the off-rate is the effective 2D onrate:

$A_{c} k_{\text {on }}=A_{c} K_{a} \times k_{r}$

$P_{a}=\frac{\left(P_{\text {measured }}-P_{\text {nonspecific }}\right)}{1-P_{\text {nonspecific }}}$,

where $P_{\text {nonspecifc }}$ and $P_{\text {measured }}$ are the nonspecific adhesion fraction and the total measured adhesion, respectively.

FRET analysis

FRET is a nonradiative process that originates from the dipole-dipole interaction between the electronic states of the donor and acceptor $(11,33)$. The energy transfer occurs only when the oscillations of the optically induced electronic coherence of the donor are resonant with the electronic energy gap of the acceptor. The efficiency of energy transfer $\left(E_{\mathrm{FRET}}\right)$ is sensitive to the distance between the donor and the acceptor, which is typically in the range of $10-100 \AA$. The energy transfer efficiency $\left(E_{\text {FRET }}\right)$ is generally given by: ${ }^{11,33}$

$E_{\mathrm{FRET}}=\frac{1}{1+\left(r / R_{0}\right)^{6}}$,

where $r$ is the distance between donor $(D)$ and acceptor $(A)$ and $R_{0}$ is Förster's distance. FRET is very sensitive to the distance between the acceptor and the donor, which may change due to conformational dynamics or other factors. The $E_{\mathrm{FRET}}$ between $D$ and $A$ was calculated by a ratiometric method to reveal the conformational dynamics using the following equation: ${ }^{11,33}$

$E_{\mathrm{FRET}}=\frac{I_{A}}{I_{A}+I_{D} \gamma}$

where $I_{A}$ and $I_{D}$ are the fluorescence emission intensities of the acceptor and the donor, respectively, and $\gamma$ is the correction factor, which is determined as the ratio of the detection efficiencies of the acceptor and donor channels according to a previously reported method. ${ }^{33}$ Before $E_{\mathrm{FRET}}$ calculation, each image was background-corrected, and the bleed-through was corrected according to a previously reported method. ${ }^{33}$ The Alexa Fluor 568 intensity was further corrected according to the scFV dissociation kinetics for FRET2 (Fig. S18).

To obtain the distribution of the FRET efficiencies, $E_{\mathrm{FRET}}$, and the corresponding distances between a FRET donor and a FRET acceptor, we tracked and measured the fluorescence intensities of single donor and acceptor molecules. For each experiment, image registration was first performed using MATLAB for the images of the corresponding frames from the donor channel and the acceptor channel. The donor and acceptor molecules were identified and tracked using TrackMate in ImageJ until the end of each FRET trajectory. The fluorescence intensities of a donor and an acceptor were measured and background-corrected frame-by-frame. Eqns. 5 and 6 were used to calculate $E_{\mathrm{FRET}}$ and the corresponding distance for both the FRET1 (Cy3/Cy5) and FRET2 (GFP/Alexa568) experiments. By tracking the trajectories of many individual FRET pairs, we obtained the values and distribution of $E_{\mathrm{FRET}}$ and the corresponding distance using Eqs. 5 and 6.

For the ensemble FRET1 experiments, because the binding affinities were different for the three pMHCs, their TCR occupancies were different. To calculate the TCR-pMHC bond distance in the bound state, the unbound pMHCs were removed from the FRET calculation. ${ }^{53}$ The bound TCRs were directly measured by Cy3/Cy5 FRET because FRET is highly distance specific ${ }^{8}$ (Fig. 1b, comparison of $\mathrm{K} 5$ with null). Only the bound pMHCs and the bound TCRs were used to calculate the FRET efficiency and distance using Eqs. 5 and 6 after background and bleed-through correction.

PMF analysis

One way to quantify the binding strength is to examine the PMF of the fluctuation of the donor-receptor distance $R$. The PMF in this context is given by: ${ }^{16,54}$

$F(R)=-k_{B} T \times \ln (P(R))$,

where $P(R)$ is the histogram representing the distance that is an average of the steady-state signals collected from 1500 independent TCR-pMHC bond trajectories. The PMF measures the free energy cost of variation for distance $R$. It is minimized at equilibrium. Its curvature governs the size of the fluctuations. A shallower potential curve implies greater fluctuation and weak binding.

Microcluster tracking analysis

For the FRET2 analysis, we developed a method to track individual $\mathrm{CD} 3 \zeta$ (donor) and TCR (acceptor) microclusters in three dimensions $(x, y$, and $z)$ using the TrackMate plugin in Fiji. ${ }^{55}$ The track for each individual donor and acceptor cluster gave the lateral movement ( $x-y$ axis) as well as the FRET2 efficiency (TCR-CD3 $\zeta$ distance, $z$-axis) as the microclusters moved toward the center and formed immunological synapses.

\section{Measurement of $\mathrm{CD} 3 \zeta$ phosphorylation}

We used phospho-flow cytometry to measure the phosphorylation of $\mathrm{CD} 3 \zeta$ at the single-cell level. ${ }^{56,57} \mathrm{CH} 27$ cells were preincubated with $10 \mu \mathrm{M}$ peptide in complete medium for $3 \mathrm{~h}$ at $37^{\circ} \mathrm{C}$. $\mathrm{CH} 27$ cells with preincubation were used as a negative control. Peptide-loaded $\mathrm{CH} 27$ cells were washed three times. ${ }^{2} 5 \mathrm{C}$. $\mathrm{C} 7 \mathrm{~T}$ cells were rested in serum-free RPMI medium at $37^{\circ} \mathrm{C}$ for $3 \mathrm{~h}$ to reduce the background phosphorylation level. ${ }^{25} \mathrm{~A}$ total of 50,000 peptide-loaded $\mathrm{CH} 27$ cells and 50,000 rested 5C.C7 T cells were precooled and mixed in a tube on ice. The tube was centrifuged at $300 \times g$ for $1 \mathrm{~min}$ at $4^{\circ} \mathrm{C}$ to initiate cell-cell contact and immediately transferred to a $37^{\circ} \mathrm{C}$ water bath to initiate T-cell stimulation. The stimulation was terminated at the indicated time points with $4 \%$ PFA fixation. After $10 \mathrm{~min}$ of fixation at room 
temperature, the cells were washed twice with ice-cold PBS containing $2 \%$ BSA and then resuspended in $80 \%$ methanol and incubated for $30 \mathrm{~min}$ at $-20^{\circ} \mathrm{C}$. After washing twice with ice-cold PBS, $0.3 \mu \mathrm{g} / \mathrm{mL}$ Alexa Flour-488-labeled anti-pY142-CD3 3 antibody (BD) was added to a final volume of $100 \mu \mathrm{L}$ of ice-cold PBS and incubated at $4{ }^{\circ} \mathrm{C}$ for $45 \mathrm{~min}$. The cells were washed three times with ice-cold PBS containing 2\% BSA and analyzed by flow cytometry. The flow cytometry data were further processed with FlowJo software.

$\mathrm{Ca}^{2+}$ imaging

For the $\mathrm{Ca}^{2+}$ flux experiments, T cells $\left(\sim 10^{6}\right)$ were incubated with $5 \mu \mathrm{M}$ of the fluorescent dye Fluo-4 AM (Thermo Fisher Scientific) for $30 \mathrm{~min}$ in complete RPMI 1640 medium. All the Fluo-4 loading and imaging experiments were performed in the presence of 2.5 $\mathrm{mM}$ probenecid. The $\mathrm{T}$ cells were washed twice with minimal imaging media (MIM; colorless RPMI with 5\% FBS and $10 \mathrm{mM}$ HEPES) and then incubated in MIM for $10 \mathrm{~min}$ at $37^{\circ} \mathrm{C}$ before data collection. ${ }^{58}$ For imaging, a LEITZ DMIRB Leica Microscope equipped with a 100× objective and an iXON Ultra 888 EMCCD camera were used. The calcium flux imaging acquisition was performed with Micromanager software. For the T cell/APC conjugate experiments, $\mathrm{CH} 27$ cells $\left(10^{6}\right)$ were incubated with 4 $\mu \mathrm{M}$ of each peptide for $4 \mathrm{~h}$ at $37^{\circ} \mathrm{C}$ and then washed with MIM. The T cells $(2 \mu \mathrm{L})$ and $\mathrm{CH} 27$ cells $(2 \mu \mathrm{L})$ were added to MIM $(300 \mu \mathrm{L})$ in the cell chamber. The chamber was sealed using mineral oil on both sides to avoid MIM evaporation. The signals from Fluo-4 were collected at intervals of $100 \mathrm{~ms}$ for up to $20 \mathrm{~min}$ and postprocessed with Fiji software.

\section{ACKNOWLEDGEMENTS}

We thank Mark M. Davis at Stanford for providing the constructs for the anti-TCR scFvs, Michael Birnbaum at MIT for providing the $\mathrm{IE}^{\mathrm{k}}$ plasmids, Xiaolei Su and Marcus Taylor in the lab of Ronald Vale at UCSF for advice regarding the preparation of the glass-supported lipid bilayer, Jillian Rosenberg for reading the manuscript, and the NIH Tetramer Core Facility for providing the PMHC monomers. This work was mainly supported by NIH grants R00Al106941 and R21Al120010, NSF CAREER Award 1653782, a Chicago Biomedical Consortium Catalyst Award (to J.H.) and postdoctoral grant PDR-092 (to D.K.S.), with additional support from the Searle Funds at The Chicago Community Trust. This work is also supported by NIH grant R01Al126756 (to E.J.A.) and R01CA022677 (to H.S.)

\section{AUTHOR CONTRIBUTIONS}

J.H. conceived, directed, and supervised the project. J.H. and D.K.S. designed the experiments. D.K.S. custom-built the fluorescence microscopy system, performed the imaging experiments, and analyzed the data unless otherwise indicated. W.F. and D.K.S. custom-built the micropipette system. W.F. performed all 2D binding kinetics assays and analyzed the data. W.F. and D.K.S. performed the $\mathrm{Ca}^{2+}$ imaging experiments and D.K.S. analyzed the data. The reagents and experimental systems were designed and tested by D.K.S. unless otherwise indicated. S.R. and E.J.A. generated the $\mathrm{IE}^{\mathrm{k}}$ proteins and made Fig. 1a. H.L. and J.Q. performed the PMF analysis. G.C. analyzed the smFRET1 data. Y.H. and H.S. prepared the CD3Z-GFP construct and transduced the primary T cells. C.C. performed the phospho-flow cytometry. D.K.S. and P.L. cultured the T cells. D.K.S. and J.H. wrote the manuscript with input from all authors.

\section{ADDITIONAL INFORMATION}

The online version of this article (https://doi.org/10.1038/s41423-019-0273-6) contains supplementary material.

Competing interests: The authors declare no competing interests.

\section{REFERENCES}

1. Irvine, D. J., Purbhoo, M. A., Krogsgaard, M. \& Davis, M. M. Direct observation of ligand recognition by T cells. Nature 419, 845-849 (2002).
2. Huang, J. et al. A single peptide-major histocompatibility complex ligand triggers digital cytokine secretion in CD4(+) T cells. Immunity 39, 846-857 (2013).

3. Huang, J. et al. The kinetics of two-dimensional TCR and PMHC interactions determine T-cell responsiveness. Nature 464, 932-936 (2010).

4. Germain, R. N. Computational analysis of $T$ cell receptor signaling and ligand discrimination-past, present, and future. FEBS Lett. 584, 4814-4822 (2010).

5. van der Merwe, P. A. \& Dushek, O. Mechanisms for T cell receptor triggering. Nat. Rev. Immunol. 11, 47-55 (2010).

6. Chakraborty, A. K. \& Weiss, A. Insights into the initiation of TCR signaling. Nat. Immunol. 15, 798-807 (2014).

7. Rudolph, M. G., Stanfield, R. L. \& Wilson, I. A. How TCRs bind MHCs, peptides, and coreceptors. Annu. Rev. Immunol. 24, 419-466 (2006).

8. Huppa, J. B. et al. TCR-peptide-MHC interactions in situ show accelerated kinetics and increased affinity. Nature 463, 963-967 (2010).

9. $\mathrm{Xu}, \mathrm{C}$. et al. Regulation of $\mathrm{T}$ cell receptor activation by dynamic membrane binding of the CD3epsilon cytoplasmic tyrosine-based motif. Cell 135, 702-713 (2008).

10. $\mathrm{Ha}, \mathrm{T}$. et al. Probing the interaction between two single molecules: fluorescence resonance energy transfer between a single donor and a single acceptor. Proc. Natl. Acad. Sci. USA 93, 6264-6268 (1996).

11. Sasmal, D. K., Pulido, L. E., Kasal, S. \& Huang, J. Single-molecule fluorescence resonance energy transfer in molecular biology. Nanoscale 8, 19928-19944 (2016).

12. Corse, E., Gottschalk, R. A., Krogsgaard, M. \& Allison, J. P. Attenuated T cell responses to a high-potency ligand in vivo. PLoS Biol. 8, e1000481 (2010).

13. Natarajan, K. et al. An allosteric site in the T-cell receptor Cbeta domain plays a critical signalling role. Nat. Commun. 8, 15260 (2017).

14. Wu, P. et al. Mechano-regulation of peptide-MHC class I conformations determines TCRantigen recognition. Mol. Cell 73, 1015-27 e7 (2019).

15. Newell, E. W. et al. Structural basis of specificity and cross-reactivity in T cell receptors specific for cytochrome c-I-E(k). J. Immunol. 186, 5823-5832 (2011).

16. Yang, $\mathrm{H}$. et al. Protein conformational dynamics probed by single-molecule electron transfer. Science 302, 262-266 (2003).

17. Choudhuri, K., Wiseman, D., Brown, M. H., Gould, K. \& van der Merwe, P. A. T-cell receptor triggering is critically dependent on the dimensions of its peptide-MHC ligand. Nature 436, 578-582 (2005).

18. Choudhuri, K. et al. Peptide-major histocompatibility complex dimensions control proximal kinase-phosphatase balance during T cell activation. J. Biol. Chem. 284, 26096-26105 (2009).

19. O’Donoghue, G. P., Pielak, R. M., Smoligovets, A. A., Lin, J. J. \& Groves, J. T. Direct single molecule measurement of TCR triggering by agonist pMHC in living primary T cells. elife 2, e00778 (2013).

20. Taylor, M. J., Husain, K., Gartner, Z. J., Mayor, S. \& Vale, R. D. A DNA-based T cell receptor reveals a role for receptor clustering in ligand discrimination. Cell 169, 108-119 e20 (2017)

21. Campi, G., Varma, R. \& Dustin, M. L. Actin and agonist MHC-peptide complexdependent $\mathrm{T}$ cell receptor microclusters as scaffolds for signaling. J. Exp. Med. 202, 1031-1036 (2005).

22. Hong, J. et al. Force-regulated in situ TCR-peptide-bound MHC class II kinetics determine functions of CD4+ T Cells. J. Immunol. 195, 3557-3564 (2015).

23. Jonsson, P. et al. Remarkably low affinity of CD4/peptide-major histocompatibility complex class II protein interactions. Proc. Natl. Acad. Sci. USA 113, 5682-5687 (2016).

24. Xiong, Y., Kern, P., Chang, H. \& Reinherz, E. T. Cell receptor binding to a pMHCII ligand is kinetically distinct from and independent of CD4. J. Biol. Chem. 276, 5659-5667 (2001).

25. Hui, E. et al. T cell costimulatory receptor CD28 is a primary target for PD-1mediated inhibition. Science 355, 1428-1433 (2017).

26. Davis, S. J. \& van der Merwe, P. A. The kinetic-segregation model: TCR triggering and beyond. Nat. Immunol. 7, 803-809 (2006).

27. Hanke, J. H. et al. Discovery of a novel, potent, and Src family-selective tyrosine kinase inhibitor. Study of Lck- and FynT-dependent T cell activation. J. Biol. Chem. 271, 695-701 (1996)

28. Yokosuka, T. et al. Newly generated T cell receptor microclusters initiate and sustain T cell activation by recruitment of Zap70 and SLP-76. Nat. Immunol. 6, 1253-1262 (2005).

29. Berg J. M., Tymoczko J. L., \& Stryer L. Biochemistry 5th edn. (W H Freeman, New York, 2002).

30. Liu, B., Chen, W., Evavold, B. D. \& Zhu, C. Accumulation of dynamic catch bonds between TCR and agonist peptide-MHC triggers T cell signaling. Cell 157, 357-368 (2014).

31. Sibener, L. V. et al. Isolation of a structural mechanism for uncoupling $T$ cell receptor signaling from peptide-MHC binding. Cell 174, 672-87 e27 (2018). 
32. Shi, X. et al. Ca2+ regulates $\mathrm{T}$-cell receptor activation by modulating the charge property of lipids. Nature 493, 111-115 (2013).

33. Roy, R., Hohng, S. \& Ha, T. A practical guide to single-molecule FRET. Nat. Methods 5, 507-516 (2008).

34. Lillemeier, B. F. et al. TCR and Lat are expressed on separate protein islands on T cell membranes and concatenate during activation. Nat. Immunol. 11, 90-96 (2010).

35. Basu, R. et al. Cytotoxic T cells use mechanical force to potentiate target cell killing. Cell 165, 100-110 (2016).

36. Altan-Bonnet, G. \& Germain, R. N. Modeling T cell antigen discrimination based on feedback control of digital ERK responses. PLoS Biol. 3, e356 (2005).

37. Gil, D., Schamel, W. W., Montoya, M., Sanchez-Madrid, F. \& Alarcon, B. Recruitment of Nck by CD3 epsilon reveals a ligand-induced conformational change essential for $\mathrm{T}$ cell receptor signaling and synapse formation. Cell 109, 901-912 (2002).

38. Guo, X. et al. Lipid-dependent conformational dynamics underlie the functional versatility of T-cell receptor. Cell Res. 27, 505-525 (2017).

39. Lee, M. S. et al. A mechanical switch couples $T$ cell receptor triggering to the cytoplasmic juxtamembrane regions of CD3zetazeta. Immunity 43, 227-239 (2015).

40. $\mathrm{Li}$, L. et al. lonic CD3-Lck interaction regulates the initiation of T-cell receptor signaling. Proc. Natl. Acad. Sci. USA 114, E5891-E5899 (2017).

41. Birnbaum, M. E. et al. Deconstructing the peptide-MHC specificity of $\mathrm{T}$ cell recognition. Cell 157, 1073-1087 (2014).

42. Howarth, M. \& Ting, A. Y. Imaging proteins in live mammalian cells with biotin ligase and monovalent streptavidin. Nat. Protoc. 3, 534-545 (2008).

43. Ozaki, C. Y. et al. Single chain variable fragments produced in Escherichia coli against heat-labile and heat-stable toxins from enterotoxigenic E. coli. PLOS ONE 10, e0131484 (2015).

44. Tsumoto, K. et al. Highly efficient recovery of functional single-chain Fv fragments from inclusion bodies overexpressed in Escherichia coli by controlled introduction of oxidizing reagent-application to a human single-chain Fv fragment. J. Immunol. Methods 219, 119-129 (1998).

45. Leisegang, M. et al. Eradication of large solid tumors by gene therapy with a T-cell receptor targeting a single cancer-specific point mutation. Clin. Cancer Res. 22, 2734-2743 (2016).

46. Su, X. et al. Phase separation of signaling molecules promotes $\mathrm{T}$ cell receptor signal transduction. Science 352, 595-599 (2016).

47. Matysik, A. \& Kraut, R. S. TrackArt: the user friendly interface for single molecule tracking data analysis and simulation applied to complex diffusion in mica supported lipid bilayers. BMC Res. Notes 7, 274 (2014).
48. Edelstein, A. D. et al. Advanced methods of microscope control using muManager software. J. Biol. Methods 1, e10 (2014).

49. Jiang, N. et al. Two-stage cooperative $\mathrm{T}$ cell receptor-peptide major histocompatibility complex-CD8 trimolecular interactions amplify antigen discrimination. Immunity 34, 13-23 (2011).

50. Sasmal, D. K., Yadav, R. \& Lu, H. P. Single-molecule patch-clamp FRET anisotropy microscopy studies of NMDA receptor ion channel activation and deactivation under agonist ligand binding in living cells. J. Am. Chem. Soc. 138, 8789-8801 (2016).

51. Lakowicz J. R. Principles of Fluorescence Spectroscopy 3rd edn. (Springer, 2006).

52. Huang, J., Edwards, L. J., Evavold, B. D. \& Zhu, C. Kinetics of MHC-CD8 interaction at the T cell membrane. J. Immunol. 179, 7653-7662 (2007).

53. Zhu, D. M., Dustin, M. L., Cairo, C. W. \& Golan, D. E. Analysis of two-dimensional dissociation constant of laterally mobile cell adhesion molecules. Biophys. J. 92, 1022-1034 (2007).

54. Chandler D. Introduction to Modern Statistical Mechanics 1st edn. (Oxford University Press, 1987).

55. Tinevez, J. Y. et al. TrackMate: an open and extensible platform for single-particle tracking. Methods 115, 80-90 (2017).

56. Dominguez, D. et al. Exogenous IL-33 restores dendritic cell activation and maturation in established cancer. J. Immunol. 198, 1365-1375 (2017).

57. Krutzik, P. O., Trejo, A., Schulz, K. R. \& Nolan, G. P. Phospho flow cytometry methods for the analysis of kinase signaling in cell lines and primary human blood samples. Methods Mol. Biol. 699, 179-202 (2011).

58. Huse, M. et al. Spatial and temporal dynamics of Tcell receptor signaling with a photoactivatable agonist. Immunity 27, 76-88 (2007).

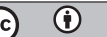

Open Access This article is licensed under a Creative Commons Attribution 4.0 International License, which permits use, sharing, adaptation, distribution and reproduction in any medium or format, as long as you give appropriate credit to the original author(s) and the source, provide a link to the Creative Commons license, and indicate if changes were made. The images or other third party material in this article are included in the article's Creative Commons license, unless indicated otherwise in a credit line to the material. If material is not included in the article's Creative Commons license and your intended use is not permitted by statutory regulation or exceeds the permitted use, you will need to obtain permission directly from the copyright holder. To view a copy of this license, visit http://creativecommons. org/licenses/by/4.0/.

(c) The Author(s) 2019 